\title{
How to quantify the temporal storage effect using simulations instead of math
}

\author{
Stephen P. Ellner*a, Robin E. Snyder ${ }^{\mathrm{b}}$ and Peter B. Adler ${ }^{\mathrm{c}}$ \\ ${ }^{a}$ Department of Ecology and Evolutionary Biology, Cornell University, Ithaca, New York \\ ${ }^{\mathrm{b}}$ Department of Biology, Case Western Reserve University, Cleveland Ohio \\ ${ }^{\mathrm{c}}$ Department of Wildland Resources, Utah State University, Logan Utah
}

Last compile: August 2, 2016

\footnotetext{
${ }^{*}$ Corresponding author. Department of Ecology and Evolutionary Biology, E145 Corson Hall, Cornell University, Ithaca NY 14853-2701. Email: spe2@cornell.edu
} 


\section{Introduction}

2

The storage effect, originally a theoretical hypothesis to explain how ecologically similar species could coexist by responding differently to environmental variability (Chesson \& Warner, 1981; Shmida \& Ellner, 1984), has developed into a core concept in community ecology (Mittelbach 2012) with empirical support from communities of prairie grasses (Adler et al., 2006), desert annual plants (Pake \& Venable, 1995; Angert et al., 2009), tropical trees (Usinowicz et al., 2012) and zooplankton (Caceres, 1997). An essential step in this maturation was mathematical analysis (Chesson, 1994, 2000a) that identified the conditions required for the storage effect to help stabilize coexistence of competitors. For the temporal storage effect, the focus of this paper, those conditions include (1) species-specific responses to environmental variability, (2) density-dependent covariance between environment and competition, and (3) buffered population growth.

A second important step was development of quantitative measures for the contribution of the storage effect to coexistence (Chesson, 1994, 2000a, 2003). These measures go beyond demonstrating that storage effect is operating, by quantifying how much it contributes to coexistence. In Angert et al. (2009), analysis of a model for competing annual plants with between-year variation in germination and growth rates led to expressions for the community-wide average storage effect in terms of quantities that could be estimated from the data, such as variance components of germination rates.

However, deriving the quantitative measures requires specialized and complicated calculations. Empirical case studies often require a new model, to capture the critical processes operating in that system, and thus a new mathematical analysis to obtain the necessary formulas (e.g. Usinowicz et al., 2012; Angert et al., 2009). This is in part because each model requires a new small-variance approximation. For example, models for competing annual plants with a seed bank were a focal example in Chesson (1994), but the first empirical application (Angert et al., 2009) required a more general model and an extensive new analysis (17 pages of online SI) to derive the necessary formulas.

Another limitation is that analytic theory is mostly limited to unstructured population models, where each species is described only by its total abundance (total number, total biomass, etc.). But demographic data are increasingly analyzed using structured population models (e.g., ma- 
trix (Caswell, 2001) and integral projection models (IPM) (Ellner et al., 2016)). Some theory for structured models is available (Dewi \& Chesson, 2003; Yuan \& Chesson, 2016), but again, empirical applications will require many different models.

Here we show how to get around these limitations through a simulation-based approach. Storage effect theory (Chesson, 1994, 2003, 2008) tells us what quantities we need to compute, specifically covariances of components of population growth rates. We show how to calculate the values by doing Monte Carlo simulations, instead of deriving model-specific formulas. The simulations can be done with any model for competing populations in which population growth is determined by competition and environmental variability. We use the Chesson \& Warner (1981) lottery model and a generic IPM to introduce ideas, and present two empirical applications: the four dominant species in Idaho sagebrush steppe (Adler et al. (2010); Chu \& Adler (2015)), and two competing algal species in a chemostat with periodic temperature variation (Descamps-Julien \& Gonzalez, 2005). These examples illustrate our approach's broad applicability.

Two types of measure for the contribution of the storage effect have been developed. The first (Chesson, 1994) comes from the "mechanistic decomposition" of low-density population growth rate into storage effect, relative nonlinearity, average response to environment, and processes operating on shorter time scales. The second (Chesson, 2003, 2008) is the "community average" measure. We focus on the first because it identifies which species benefit from a storage effect, but the community average measure can also be calculated by our methods (see SI section Section SI.3).

\section{Storage effect theory}

Our approach is based on two key concepts from storage effect theory (Chesson, 1989, 1994, 2000a), which we now review.

Storage effect theory assumes that the instantaneous population growth $r(t)$ for each species $j$ (see Box 1) can be written as a function of an environment-dependent parameter $E_{j}(t)$ and the competitive pressure $C_{j}(t) . \quad r_{j}$ is assumed to be an increasing function of $E_{j}$, and a decreasing function of $C_{j}$. In the lottery model (Chesson \& Warner, 1981) $E_{j}$ is the per-capita fecundity of species $j$ adults, and $C_{j}$ is the number of new offspring in all species divided by the number of open sites. 
The first key concept is that a storage effect occurs, and stabilizes coexistence, when a rare species escapes the damaging effects of $E C$ covariance, i.e. covariance between environment and competition. Consider a two species community. Stable coexistence occurs if each species has a positive average population growth rate as an invader, facing the other species as resident (at relative abundance near 1). For storage effect to occur, $E C$ covariance has to hurt a resident: when the resident has a good- $E$ year, its competition $C$ tends to be above-average, limiting population growth. This is a reasonable expectation, because a common species can't avoid intraspecific competition (Fig. 1). But a rare invader may not have that problem, so that it can increase rapidly when it has a good year.

But the invader also has bad years: poor environment and possibly high competition because the resident is doing well. To increase in the long run, its population growth rate $r$ must be "buffered" against large decreases in bad years (this is sometimes called subadditivity). This occurs when the impact of competition is weaker (less negative) in bad years than in good years:

$$
\frac{\partial}{\partial E}\left(\frac{\partial r}{\partial C}\right)=\frac{\partial^{2} r}{\partial E \partial C}<0 .
$$

This is equivalent to the definition (Chesson, 1994, eqn. 14) in terms of "standard" environment and competition parameters (see Section SI.2).

Combining subadditivity with density-dependent $E C$ covariance gives the situation in Fig. 1, where the invader's average population growth rate exceeds the resident's. This difference is stabilizing because it benefits whichever species is rare at the time.

The second key concept (Chesson, 1994) is that the storage effect can be quantified by asking, for each of the $M \geq 2$ species in a community: how much does $E C$ covariance contribute to the difference between its population growth rate as an invader, and the population growth rates of the resident species? Specifically, storage effect for species $i$ is defined (Chesson, 1994, eqn. 22) to be the contribution of $E C$ covariance to the difference between the invader and resident growth rates,

$$
\bar{r}_{i}\left(E_{i \backslash i}, C_{i \backslash i}\right)-\sum_{r \neq i} q_{i r} \bar{r}_{r}\left(E_{r \backslash i}, C_{r \backslash i}\right) .
$$


Here $\bar{r}$ denotes average population growth rate when the community is at its stochastic steady state, and $j \backslash k$ indicates a value for species $j$ when species $k$ is absent, e.g. $E_{i \backslash i}$ is $E(t)$ for species $i$ when invading the community. The sum runs over all resident species, indexed by $r .{ }^{1}$ The scaling factors $q_{i r}$, which determine how each resident is weighted relative to the invader, measure the relative sensitivity to competition of invading species $i$ and resident species $r$ (see SI section Section SI.5 for the precise definition and methods to calculate them). The analytic theory shows that these factors define the appropriate weighting so that invader population growth rate can be separated into components that "measure the contributions of different coexistence-affecting mechanisms" (Chesson, 1994, p. 241).

\section{The simulation-based method}

This paper shows how quantitative storage effect measures can be computed through simulations with a model for competing species. We are not dispensing with previous theory; we just diverge from it in using Monte Carlo simulations, instead of analytic small-variance approximations, to obtain numerical values for the measures.

The central idea is to compute each growth rate, $\bar{r}$, in equation (2) twice for each species, by simulating the model with and then without $E C$ covariance. The difference between the values of (2) calculated from the two simulations is then the contribution of $E C$ covariance to the value of (2), which is exactly the definition of the storage effect in the analytic theory (Chesson, 1994, p.240). This simulation-based comparison does not use small-variance approximations, so it is more general than previous analytic approaches, and potentially more accurate because the error converges to zero as simulation length increases.

To explain the procedures, we use the classic lottery model (Chesson \& Warner, 1981); complete $\mathrm{R}$ code for the calculations is in Table SI-1. We use this model to give a clear example of our approach, even though the storage effect for this model has been found analytically (for small variance). For simplicity we consider two species with equal, constant death rates. In the model, the habitat consists of $N$ sites that, at each annual census, are each occupied by one female adult of either species 1 or species 2 . Thus $N_{1}(t)+N_{2}(t) \equiv N$, where $N_{i}$ is the number of sites occupied by

\footnotetext{
${ }^{1}$ Using a subscript $r$ to index resident species is potentially confusing, but this notation is standard in storage effect theory so it would be more confusing to do something else.
} 
species $i$. Following the census each adult produces $B_{i}(t)$ juveniles. The adult per-capita fecundities $B_{1}(t)$ and $B_{2}(t)$ are random variables, representing the effects of environmental fluctuations. A fraction $\delta$ of adults in each species then dies, leaving $\delta N$ open sites. Competition among juveniles is neutral, so that a fraction $B_{1} N_{1} /\left(B_{1} N_{1}+B_{2} N_{2}\right)$ of open sites are occupied by species 1 , the rest by species 2 . By time $t+1$ these new recruits have become adults. The resulting population dynamics are

$$
N_{i}(t+1)=(1-\delta) N_{i}(t)+\delta N \frac{B_{i}(t) N_{i}(t)}{B_{1}(t) N_{1}(t)+B_{2}(t) N_{2}(t)}, \quad i=1,2 .
$$

The model is completed by specifying distributions for the $B_{i}$ (we assume lognormal distributions with possibly nonzero correlation between $B_{1}(t)$ and $B_{2}(t)$ ) and parameter values (lines xx-yy in Box SI-1).

The steps to calculate the storage effect for species 1 are as follows.

(Step 1) First, define the environmental variable $E$ and competition $C$. We set $E_{i}=B_{i}$, and $C_{1}=C_{2}=\left(B_{1} N_{1}+B_{2} N_{2}\right) /(\delta N)$, the ratio between the number of competing juveniles and the number of available sites. We then have

$$
r_{i}(t)=\log \left(1-\delta+E_{i}(t) / C_{i}(t)\right)
$$

$E$ and $C$ can be defined in other ways: (Chesson $(1989,1994)$ uses the $\log$ of our $E$ and $C$ ) but this has no effect on results.

(Step 2) The second step is to generate the environment sequence $E(t)=\left(E_{1}(t), E_{2}(t)\right)$ and a second independent environment sequence $E^{\#}(t)=\left(E_{1}^{\#}(t), E_{2}^{\#}(t)\right)$, for $t=0$ to some large time $T .^{2}$ For the lottery model we use the multivariate Gaussian random number generator mvrnorm to create $\log B_{1}(t)$ and $\log B_{2}(t)$ series, and do the same again to make $\log B_{1}^{\#}(t)$ and $\log B_{2}^{\#}(t)$. An alternative, which is sometimes simpler, is to make $E^{\#}(t)$ by shuffling $E(t)$ at random. Both methods have the necessary effect: $E$ and $E^{\#}$ are independent of each other but have the same marginal distribution.

\footnotetext{
${ }^{2} E^{\#}$ is pronounced "E - sharp".
} 
(Step 3) The third step is using $E_{1}(t), E_{2}(t)$ to do a long simulation of the model with species 1 as an invader - at zero density or too rare to affect other species (e.g., relative abundance below $\left.10^{-8}\right)$. At each time step, compute and save the population growth rate of each species, $r_{j}(t)=\log \left(N_{j}(t+1) / N_{j}(t)\right), j=1,2$ where $N_{j}$ is total population size of species $j$ (or total biomass, total cover, etc., depending on the model's units). At the same time, use $E_{1}^{\#}(t), E_{2}^{\#}(t)$ to compute what the population growth rates would be with these different values of the environmentdependent parameters, all else being equal (including the abundance and population structure of each species): call these $r_{j}^{\#}(t)$.

In the lottery model, with species 1 invading and species 2 resident we have $C_{1}(t)=C_{2}(t)=$ $B_{2}(t) N /(\delta N)=B_{2}(t) / \delta$. We substitute these into equation (4) to compute the population growth rates,

$$
r_{1}(t)=\log \left(1-\delta+\delta B_{1}(t) / B_{2}(t)\right),
$$

$$
r_{1}^{\#}(t)=\log \left(1-\delta+\delta B_{1}^{\#}(t) / B_{2}(t)\right) .
$$

Only $B_{1}$ is "sharped" in $r_{1}^{\#}$, because $\delta / B_{2}(t)$ in that formula is $C_{1}(t)$, which is carried over from the first simulation. This is typical: because the $C$ (competition) for a species is often a function of the $E_{\mathrm{S}}$ (environments) for several species, formulas for $r^{\#}$ often include $E \mathrm{~s}$ and $E^{\#} \mathrm{~S}$ (here, $B$ and $\left.B^{\#}\right)$. For species 2,

$$
\begin{aligned}
r_{2}(t) & =\log \left(1-\delta+\delta B_{2}(t) / B_{2}(t)\right), \\
r_{2}^{\#}(t) & =\log \left(1-\delta+\delta B_{2}^{\#}(t) / B_{2}(t)\right) .
\end{aligned}
$$

(Step 4) Next, we compute the average population growth rates

$$
\bar{r}_{j}=\mathbb{E}\left[r_{j}(t)\right], \quad r_{j}^{\#}=\mathbb{E}\left[r_{j}^{\#}(t)\right], j=1,2
$$

where $\mathbb{E}$ denotes the average (i.e., expectation) over the simulation. In general, to eliminate effects of initial transients, a burn-in period should be omitted (e.g., average $r(t)$ and $r^{\#}(t)$ over times $t=500$ to $T$ ). The resident species 2 necessarily has $\bar{r}_{2}=0$, but computing it is a useful check on your code. 
(Step 5) Next, find the scaling factor $q_{12}$. In the symmetric lottery model with equal death rates $q_{12}=q_{21}=1$ (Chesson, 1994). In our other case studies the scaling factors are not known, and we explain how they can be calculated by simulation.

(Step 6) Finally, the storage effect for species 1 is (by definition) the change in the value of eqn. (2) when $E C$ covariance is removed. This is

$$
\Delta I_{b, 1}=\left(\bar{r}_{1}-q_{12} \bar{r}_{2}\right)-\left(r_{1}^{\#}-q_{12} r_{2}^{\#}\right)=\bar{r}_{1}-r_{1}^{\#}+q_{12} r_{2}^{\#},
$$

(note $\bar{r}_{2}=0$ because species 2 is the resident). The subscript $b$ in $\Delta I_{b, 1}$ stands for "betweenspecies", because this measure compares the focal species as invader with others as residents. The community average storage effect measure is a sum of terms comparing each species in resident and invader states (see Angert et al. (2009, SI eqn. 6), Chesson (2008, Table 6.3)). These terms can also be calculated using our methods (Section SI.3).

The parameters chosen in Table SI-1 give species 1 a competitive disadvantage, lower mean fecundity. Running the code gives

$$
\bar{r}_{1}=0.031, r_{1}^{\#}=0.082, r_{2}^{\#}=0.114, \Delta I_{b, 1}=0.06
$$

Species 1 persists $\left(\bar{r}_{1}>0\right)$, but because $\bar{r}_{1}<\Delta I_{b, 1}$ we know that it persists because the storage effect overcomes its competitive disadvantage.

An interesting case is complete symmetry, meaning that $B_{1}(t)$ and $B_{2}(t)$ have the same marginal distributions. Then $B_{1}^{\#}(t) / B_{2}(t)$ and $B_{2}^{\#}(t) / B_{2}(t)$ are identically distributed, so $r_{1}^{\#}=r_{2}^{\#}$ and therefore $\Delta I_{b, 1}=\bar{r}_{1}$. This says that a positive low-density growth rate of species 1 (when it occurs) is entirely due to the storage effect, which is true because storage effect is the only potential stabilizing mechanism in the completely symmetric lottery model.

The storage effect for species 2 is estimated the same way: simulate with species 2 invading and species 1 resident, and calculate

$$
\Delta I_{b, 2}=\bar{r}_{2}-r_{2}^{\#}+q_{21} r_{1}^{\#}
$$

(note that all the $r_{j}$ s in (8) are $r_{j \backslash 1}$, while all those in (9) are $r_{j \backslash 2}$ ). 
The steps in our approach are summarized in Box 2. We now present several case studies which show how to implement those steps in other settings: continuous time, periodic environmental variation, structured populations, and three or more competing species.

\section{Example: algal coexistence in a periodic environment}

Storage effect theory and empirical applications have emphasized between-year variability, but within-year variation can also promote coexistence (Brown, 1989a,b; Chesson et al., 2001; Mathias \& Chesson, 2013). Even periodic (e.g., seasonal) variation can maintain coexistence, in both theory (Stewart \& Levin, 1973; Smith, 1981; Brown, 1989a; Smith \& Waltman, 1995; Mathias \& Chesson, 2013) and experiments (e.g., Sommer, 1984, 1985; Descamps-Julien \& Gonzalez, 2005), supporting G.E. Hutchinson's proposal that the "paradox of the plankton" might be explained by environmental variability that favors different species at different times.

However, none of these empirical examples quantify the storage effect's contribution to coexistence. For example, Descamps-Julien \& Gonzalez (2005) demonstrated coexistence of competing diatom species in a chemostat with periodic temperature variation. Having no way to quantify the storage effect, Descamps-Julien \& Gonzalez (2005) argued that the requirements for the storage effect were satisfied (e.g., "the compensatory dynamics indicate the strong covariance between the environment and interspecific competition", p. 2823), and that relative nonlinearity of competition (Chesson, 1994, 2000b) could be ruled out as a coexistence mechanism because they did not observe "endogenously generated resource fluctuations" (p. 2822). However, relative nonlinearity can also occur when populations fluctuate in response to an exogenous factor (eqn. 6 in Chesson, 2000b; Yuan \& Chesson, 2015). Other experiments on competition in periodic environments share the problem that the contribution of the storage effect could not be quantified. Here we show how this can be done, using the Descamps-Julien \& Gonzalez (2005) experiments and model.

The Descamps-Julien \& Gonzalez (2005) model is a standard two-species chemostat, with temperature-dependent parameters for resource uptake and reproduction:

$$
\frac{d S}{d t}=D\left(S_{0}-S\right)-Q_{1} x_{1} \frac{V_{1} S}{K_{1}+S}-Q_{2} x_{2} \frac{V_{2} S}{K_{2}+S}
$$

$$
\frac{d x_{j}}{d t}=x_{j} \frac{V_{j} S}{K_{j}+S}-D x_{j}, \quad j=1,2 .
$$


$S$ is extracellular silicate concentration in the chemostat, $x_{i}$ are population densities of the diatoms, Cyclotella pseudostelligera and Fragilaria crotonensis. $S_{0}$ is silicate concentration in the inflow, and $D$ is dilution (outflow) rate. Parameters $V_{j}$ (maximum reproduction rate), $K_{j}$ (half-saturation constant), and $Q_{j}$ (resource required to produce one individual) all depend on temperature

$$
\theta(t)=\theta_{0}+a \sin (2 \pi t / P)
$$

which is periodic with mean $\theta_{0}$, amplitude $a$, period $P$. Functions specifying how $Q_{j}, V_{j}$ and $K_{j}$ depend on temperature were estimated from batch experiments (Fig. 2). Predictions from this model match microcosm experiments (Descamps-Julien \& Gonzalez, 2005) which found coexistence under fluctuating temperatures $\left(\theta_{0}=18^{\circ} \mathrm{C}, a=6, P=60 \mathrm{~d}\right)$ but not constant temperature; see Fig. SI-1.

In a continuous-time model, average population growth $\bar{r}_{j}$ is $\frac{1}{T} \int_{0}^{T} r_{j}(\tau) d \tau$ in the limit $T \rightarrow \infty$, which can be evaluated by averaging over finely-spaced times $t_{k}=\frac{k T}{m}$ with $T \gg 1, m \gg T$ :

$$
\mathbb{E}\left[r_{j}\right] \approx \frac{1}{m+1} \sum_{k=0}^{m} r_{j}\left(E\left(t_{k}\right), C\left(t_{k}\right)\right)
$$

$T$ and $m$ must be large enough (in practice this means that doubling their values has negligible effect), and the system should be in steady state at $t=0$ (i.e., $t=0$ is after the actual start of the experiment or simulation).

Step 1 is defining $E$ and $C$ to match the concept of the storage effect in Fig. 1. $E$ should represent potential population increase, and $C$ the extent to which increase is limited by competition. We therefore set $E_{j}(t)=V_{j}(t)$, and $C_{j}(t)=\left(K_{j}(t)+S(t)\right) / S(t)$ so that

$$
r_{j}\left(E_{j}, C_{j}\right)=\frac{1}{x_{j}} \frac{d x_{j}}{d t}=\frac{E_{j}}{C_{j}}-D
$$

Our definition of $C$ follows Freckleton et al. (2009), who argued for measuring competition by the ratio between potential and achieved performance. In a similar model Mathias \& Chesson (2013) define $C$ so that $r=E(1-C)-D$ but both definitions give the same results in our approach.

For Step 2, eqn. (11) combined with the temperature-dependent maximum uptake rate $V_{j}$ gives $E_{j}\left(t_{k}\right)=V_{j}\left(\theta\left(t_{k}\right)\right)$ for both species. We create $E^{\#}\left(t_{k}\right)$ by shuffling at random the $E\left(t_{k}\right)$ 
values in eqn. (12). This destroys temporal autocorrelation in $E$, not just covariance with $C$. However, correlation in $E^{\#}$ has no effect on $\mathbb{E}\left[r_{i}\left(E^{\#}, C\right)\right]$ so long as $E^{\#}$ and $C$ are independent. ${ }^{3}$ Any shuffling that makes $E^{\#}$ independent of $C$ can therefore be used.

For Step 3 we run a long baseline simulation using $E(t)$ (or do a long experiment) with species 1 as invader $\left(x_{1}(t)=0\right)$, computing and saving $r_{1 \backslash 1}\left(t_{k}\right)=r_{1}\left(E\left(t_{k}\right), C_{1}\left(t_{k}\right)\right)$ and $r_{2 \backslash 1}\left(t_{k}\right)=$ $r_{2}\left(E\left(t_{k}\right), C_{2}\left(t_{k}\right)\right)$ using (13). At each time $t_{k}$ we also compute $r_{1 \backslash 1}^{\#}\left(t_{k}\right)$ and $r_{2 \backslash 1}^{\#}\left(t_{k}\right)$ by using $E^{\#}(t)$ in place of $E(t)$. Averaging the saved $r$ values (Step 4) gives $\bar{r}_{1 \backslash 1}, \bar{r}_{2 \backslash 1}, r_{1 \backslash 1}^{\#}, r_{2 \backslash 1}^{\#}$. Repeating with species 2 as invader gives $\bar{r}_{1 \backslash 2}, \bar{r}_{2 \backslash 2}, r_{1 \backslash 2}^{\#}, r_{2 \backslash 2}^{\#}$.

Step 5 is to compute the scaling factors $q_{i r}$, which have not been derived for this model. The $q_{i r}$ are defined (Chesson, 1994) in terms of the competitive effects $\mathscr{C}$ experienced by each species when species $i$ is invader and all others (indexed by $r$ ) are resident. Define

$$
\mathscr{C}_{j}=-r_{j}\left(E_{j}^{*}, C_{j}\right)
$$

where the baseline environment $E_{j}^{*}$ should be near a central value of $E_{j}(t)$ such as the mean or median. $\mathscr{C}_{j}>0$ when competition $C_{j}$ is strong enough that the population would decrease in the baseline environment. Then for invading species $i$ and resident species $r$,

$$
q_{i r}=\frac{\partial \mathscr{C}_{i \backslash i}}{\partial \mathscr{C}_{r \backslash i}}
$$

evaluated at the $C_{r}$ where $\mathscr{C}_{r \backslash i}=0$.

We can't easily calculate the derivative in eqn. (15) analytically, but we can find its value using the simulation with species 1 invading species 2 . Define $E_{1}^{*}=E_{2}^{*}=$ average temperature over the simulation. At each time $t_{k}$, we compute and save $\mathscr{C}_{j \backslash 1}(t)=-r_{j}\left(E_{j}^{*}, C_{j}(t)\right), j=1,2$ calculated from (13): what population growth would be if $E_{j}$ was at $E_{j}^{*}$. Plotting the $\mathscr{C}_{1 \backslash 1}\left(t_{k}\right)$ values as a function of $\mathscr{C}_{2 \backslash 1}\left(t_{k}\right)$ (Fig. 2D) traces out their relationship. To evaluate the derivative in (15), we fit a nonlinear regression curve, and $q_{12}$ is the slope of the regression curve at $\mathscr{C}_{2 \backslash 1}=0$. Repeating

\footnotetext{
${ }^{3}$ Write $\mathbb{E}\left[r\left(E^{\#}, C\right)\right]=\iint r(x, y) p_{E^{\#}, C}(x, y) d x d y$ where $p_{E^{\#}, C}$ is the joint density function of $E^{\#}$ and $C$. When $E^{\#}$ and $C$ are independent, $p_{E^{\#}, C}(x, y)=p_{E \#}(x) p_{C}(y)$. Because $E^{\#}$ is a reshuffling of $E, p_{E \#}=p_{E}$. We therefore have $\mathbb{E}\left[r\left(E^{\#}, C\right)\right]=\iint r(x, y) p_{E}(x) p_{C}(y) d x d y$ for any reshuffling that makes $E^{\#}$ and $C$ independent.
} 
this process with the roles swapped gives $q_{21}$. Finally (Step 6) we compute $\Delta I$ by substituting the calculated $\bar{r}, r^{\#}$ and $q_{i r}$ values into equations (8) and (9).

The results (Table 1) show that although temperature fluctuations are necessary for coexistence, the storage effect contribution is small, especially for Cyclotella. Over the experiment's temperature range $\left(12-24^{\circ} \mathrm{C}\right)$ Fragillaria is affected little by temperature, so when it is sole resident, $S$ remains low, $C_{1}$ and $C_{2}$ are nearly constant, and so $E C$ covariance $\chi \approx 0$ for both species. Because $E C$ covariance has little effect on either species, $\Delta I_{b} \approx 0$ for the invader, Cyclotella. In contrast, $S$ varies when Cyclotella is resident (in model simulations and the experiments), and Cyclotella is limited by $E C$ covariance $\left(\chi_{r}=0.17\right)$ : when temperature is favorable, silicate is quickly depleted (see online SI Fig. SI-2). At the same time, Fragillaria as invader has little EC covariance because its $E$ is nearly constant. Consequently $\Delta I_{b}>0$ for Fragillaria, because the negative impact of EC covariance on Cyclotella as resident contributes to the growth rate advantage of Fragillaria as invader.

However, even without the storage effect contribution, Fragillaria's low-density growth rate would be positive (i.e., $\bar{r}_{i}>\Delta I_{b}$ ). The same is true for Cyclotella. Coexistence requires environmental fluctuations - at any constant temperature only one species persists - but the storage effect cannot be acting alone to maintain coexistence, as both invader growth rates are positive without it.

Environment and resource fluctuations can also affect population growth rates through nonlinear averaging. In this model variability in $S$ is the only source of nonlinear averaging, because $\mathbb{E}\left[r_{i}\right]$ is linear in $V_{i}$, and the $K_{i}$ are constant over the experiment's temperature range. We can quantify the nonlinear averaging effect by comparing population growth rates from a "flattened" simulation in which $S$ is held constant at its average value, with population growth rates from the baseline simulation in which $S$ fluctuates. The flattened simulations remove the storage effect (because $\operatorname{Cov}(E, C)=0$ when $C$ is constant) and also nonlinear averaging, so the differences $r^{b}-r^{\#}$, for each species in resident and invader states, measure the effect of nonlinear averaging. Nonlinear averaging is unimportant when Fragillaria is resident, but when Cyclotella is resident and $S$ is variable, the effects of nonlinear averaging on the species are much larger than the storage effect.

Because of a contamination problem, the Descamps-Julien \& Gonzalez (2005) experiments provide reliable data for only one cycle of temperature variation. Our analysis here therefore uses 
model simulations. However, exactly the same calculations can be done with data from a long experiment, sampled frequently enough to capture the population fluctuations.

\section{Structured Populations}

We now use a hypothetical "prototype" IPM to illustrate how our approach works with structured populations, and then analyze an empirically-parameterized IPM for sagebrush steppe.

Our prototype IPM has the typical structure in which demographic rates are functions of logtransformed size $z$ (e.g. Ellner \& Rees, 2006; Coulson, 2012). The model also includes timevarying environmental responses, and an interaction between environment and competition to allow a storage effect in the model.

Survival of species $j$ is described by logistic regression,

$$
\operatorname{logit} s_{j}(z, t)=b_{0, j}^{(S)}+b_{1, j}^{(S)} z+b_{2, j}^{(S)} E_{j}(t)-\left[\sum_{k=1}^{2} \alpha_{j k}^{(S)} N_{k}(t)+\sum_{k=1}^{2} \beta_{j k}^{(S)} E_{k}(t) N_{k}(t)\right] \text {, }
$$

where $E_{j}$ is the environment covariate for species $j$ in year $t$ (representing a measured variable, such as rainfall, affecting all demographic rates), $N_{j}(t)=\int e^{z} n_{j}(z, t) d z$ is total cover of species $j$ in year $t$ (because $z$ is the log of individual cover). The term in brackets is the $C_{j}(t)$ for survival (Step 1). Similarly, for growth we assume that each individual's size at time $t+1$, conditional on its size at time $t$, is Gaussian with constant variance, and mean given by the right-hand side of (16) with coefficients $b_{0, j}^{(G)}$ and so on. Per-capita fecundity $B_{j}(z, t)$ is modeled with Poisson regression using the canonical $\log$ link function, so that $\log B_{j}(z, t)$ equals the right-hand side of (16) with coefficients $b_{0, j}^{(F)}$ and so on.

For Step 2, environment covariates $E_{j}(t)$ and then $E_{j}^{\#}(t)$ for each year are drawn from lognormal distributions with specified means and variance-covariance matrices ("distribution sampling", Metcalf et al., 2015).

Population structure introduces two new aspects in Steps 3 and 4 of our approach. First, survival, growth, and fecundity are separate process, so $\vec{C}_{i}$ is now a vector of the distinct $C$ s for survival, growth and fecundity. Second, population growth rates depend on population structure, so $r^{\#}$ calculations use the population structures from the corresponding baseline simulation. So if 
$K_{j}\left(E_{j}, \vec{C}_{j}\right)$ is the projection kernel for species $j$, then

$$
r_{j}^{\#}(t)=\log \left(N_{j}^{\#}(t+1) / N_{j}(t)\right)
$$

$$
\text { where } \quad N_{j}^{\#}(t+1)=\int e^{z} n_{j}^{\#}(z, t+1) d z, \quad n_{j}^{\#}(t+1)=K_{j}\left(E_{j}^{\#}(t), \vec{C}_{j}(t)\right) n_{j}(t) \text {. }
$$

As always, $\vec{C}_{j}$ is from the baseline simulation, and depends on $E_{j}$ but not $E_{j}{ }^{\#}$. Scaling factors were estimated by the regression method (Step 5c), as in Fig. 2D (see SI script IPM-qir-wrapper.R).

Fig. 3 shows results for completely symmetric parameters $\left(b_{0,1}^{(S)}=b_{0,2}^{(S)}, \beta_{11}=\beta_{12}=\beta_{21}=\right.$ $\beta_{12}=\beta_{E N}$ etc.); the only difference between species is that they respond to different environment covariates having identical marginal distributions. In all cases the storage effect goes to zero as $\operatorname{Cor}\left(E_{1}, E_{2}\right)$ increases to 1 , as expected: nobody ever escapes $E C$ covariance because a good- $E$ year is good for everyone and competition is high. Similarly, the storage effect is zero when $\beta_{E N}=0$ because nobody ever experiences $E C$ covariance. Fluctuating fecundity produces a stabilizing (positive) storage effect (fig. 3A), as in the lottery model, whereas fluctuating growth does not (fig. 3B). Storage effect from fluctuating survival can be positive (fig. 3C,D) depending on whether parameter values make mean survival high or low. This contrasts with the lottery model, where variable survival can only stabilize coexistence when survival is high and correlated with recruitment fluctuations (Chesson \& Warner, 1981).

However, these results are largely dictated by the model's structure. The linear predictors in the demographic models (e.g., the right-hand side of (16)) are additive in $E$ and $C$. Consequently, the nonlinearities that can buffer populations against poor years via subadditivity (or amplify the decrease in poor years via superadditivity) are all produced by the link function, which specifies how the mean response depends on the linear predictor in a generalized linear model. Specifically (see section Section SI.6), a positive storage effect is only possible if the inverse of the link function is concave up. For fecundity, the inverse link is the exponential function: the storage effect can be positive. For survival the inverse link is the logistic function, which is concave up for survival below 0.5 , so the storage effect can be (and is) positive in our model, and concave down for survival above 0.5 , so the storage effect has to be negative. For growth the inverse link is the identity (zero concavity) so the storage effect is near 0 . Our results for this model are a cautionary tale: effects 
of environmental variability are mediated by second derivatives, and those are often dictated by statistical "habits" that are harmless for other purposes (e.g., projecting population growth).

\section{Empirical four-species IPM}

Our empirical IPM is closely based on the Chu \& Adler (2015) model for the dominant species in a sagebrush steppe community, three perennial grasses and the shrub, Artemisia tripartita. Environmental variation was modeled by fitted random year effects ("kernel resampling", Metcalf et al., 2015). However, Chu \& Adler (2015) assumed constant competition coefficients, hence $C$ is not a function of $E$, precluding $E C$ covariance. Even if a storage effect were present in the natural system, the model could not generate one.

We therefore re-fitted the model with temporal variation in interaction coefficients, fitted as random year effects (see SI section Section SI.7), so that a storage effect is possible. The linear predictors are then

$$
b_{0, j}+b_{1, j} z+b_{2, j} E_{j}(t)-\left[\sum_{k=1}^{4} \alpha_{j k} W_{j k}(t)+\sum_{k=1}^{4} D_{j k}(t) W_{j k}(t)\right]
$$

where $W_{j k}$ is the competitive pressure from species $k$ on species $j$. The crucial difference from Chu \& Adler (2015) is that $C$ (the term in brackets) has random year effects $D_{j k}$ so that $E C$ covariance can occur. The difference with the prototype IPM (16) is that the year effects $E_{j}$ and $D_{j k}$ are distinct, so $E C$ covariance only occurs if the fitted $E$ and $D$ s for a species are correlated.

With multiple species, the storage effect for species $i$ is

$$
\Delta I_{b, i}=\bar{r}_{i \backslash i}-r_{i \backslash i}^{\#}+\sum_{r \neq i} q_{1 r} r_{r \backslash i}^{\#}
$$

The random variation in interaction strengths made it difficult to estimate scaling factors by regression, so we used an alternate approach based on species' responses to perturbed competitor densities (Step 5d - see sect. Section SI.5(d)). Otherwise, everything is the same as with the prototype IBM.

The results (Table 2) are very consistent: the storage effect is tiny for all species and all demographic processes, separately or together. This occurs because $E C$ covariance is so low, for the empirically fitted parameters, that removing it has essentially no effect and there are only 
minute differences between each $\bar{r}$ and the corresponding $r^{\#}$ (tabulated in section Section SI.8). When environmental variability is completely removed, one species, Artemisia, declines slowly to extinction in the model (Adler et al., 2010). As in the chemostat study above, some fluctuationdependent mechanism besides the storage effect must be contributing to persistence of Artemisia.

\section{Discussion}

Until now, empirical applications of temporal storage effect theory had to begin by analyzing a community model to derive formulas for the storage effect and other mechanisms in terms of measurable attributes. Our simulation-based approach works directly with a parametrized model for competing species, without requiring model-specific mathematical analysis, and can give more accurate results than small-variance approximations. We have shown how our approach can be used in practice with a wide range of models, using the same kinds of data as analytic approaches.

Our empirical examples highlight the fact that the storage effect is only one component of low-density growth rates. Simulation-based approaches can and should be developed for the other fluctuation-dependent stabilizing mechanism, relative nonlinearity (Chesson, 1994), as well as mechanisms based on spatial variation, the spatial storage effect (Melbourne and Shoemaker in prep), fitness-density covariance, and their interactions with temporal variability (Chesson, 2000a). We have considered only competition, either direct or through resource competition. Coexistence can also be mediated by other interactions: shared enemies, mutualists, facilitation, etc., and we need methods to quantify their stabilizing effects. Simulation methods are also needed to quantify the overall contributions of stabilizing and equalizing mechanisms, and the stabilizing and equalizing components of each mechanism. All these methods should accommodate structured populations.

Our case studies highlight the importance of thinking carefully about model structure, because "traditional" choices can have side-effects that make it impossible or difficult for a storage effect to operate. For example, IPMs often include main effects (in the ANOVA sense) of competition and environmental stochasticity, but not their interaction, in the linear predictor of demographic models. A main motivation for this paper was our experience of fitting a traditional IPM to Kansas grassland data we had previously studied (Adler et al., 2006) using a spatially explicit individualbased model (IBM). The IBM revealed that environmental variability was important for species coexistence; the IPM said that environmental variability played no role (Chu \& Adler, 2015) - 
as an unintended side-effect, we eventually realized, of structural assumptions in its demographic models. The methods here will let us re-visit the Kansas data with an IPM that can include the storage effect and other mechanisms.

Similarly, demographic modelers have not given much attention to estimating second derivatives, but effects of environmental variability are mediated by the second derivatives (curvature) of demographic responses to environmental factors. The optimal statistical model for predicting a response is generally not optimal for predicting its derivatives (Fan \& Gijbels, 1996). Standard practices such as logistic regression should be supplemented by checking robustness to more flexible approaches such as generalized additive models, and by statistical tests for curvature (Ye and Hooker, in prep).

The scaling factors $q_{i r}$, measuring relative sensitivity to competition, are the most difficult and delicate piece in storage effect theory. They are needed when mechanisms are quantified by comparing each species as invader with other species as residents. The $q_{i r}$ are well-defined when competitive impacts on an invader (eqn. 14) are a unique function of the impacts on residents. But that is not always true (see sect. Section SI.5), our empirical IPM being an example. However, as Chesson (2008, p.151) noted, often a mechanism "is most easily understood in terms of how the conditions encountered by an individual species change between its resident and invader states." This corresponds to the Adler et al. (2007) characterization of stabilizing mechanisms: "species' per capita growth rates decline as their relative abundance or frequency in a community increases". Our approach should make it possible to quantify stabilizing mechanisms from this more intuitive perspective, in which scaling factors are not needed because each species is compared to itself at a different abundance. Instead, measures will be calculated from specific effects of falling to low relative abundance - for example, by asking what population growth rate would be if $E C$ correlation were unaffected by becoming rare.

"Modern coexistence theory" is a conceptually powerful framework that has become central to community ecology. The analytic theory is essential for understanding how different coexistence mechanisms arise and interact. But there are still very few examples of carrying the theory into the field in a rigorous, quantitative way. We hope that the tools introduced here, and the potential extensions that we suggest, will change this situation. 


\section{References}

Adler, P.B., Ellner, S.P. \& Levine, J.M. (2010). Coexistence of perennial plants: an embarrassment of niches. Ecology Letters, 13, 1019-1029.

Adler, P.B., HilleRisLambers, J., Kyriakidis, P., Guan, Q. \& Levine, J.M. (2006). Climate variability has a stabilizing effect on coexistence of prairie grasses. Proc. Nat. Acad. Sci. U. S. A., 103, 12793-12798.

Adler, P.B., HilleRisLambers, J. \& Levine, J.M. (2007). A niche for neutrality. Ecology Letters, 10, 95-104.

Angert, A.L., Huxman, T.E., Chesson, P. \& Venable, D.L. (2009). Functional tradeoffs determine species coexistence via the storage effect. Proceedings of the National Academy of Sciences (USA), $106,11641-11645$.

Brown, J.S. (1989a). Coexistence on a seasonal resource. American Naturalist, 133, 168-182.

Brown, J.S. (1989b). Desert rodent community structure: a test of four mechanisms of coexistence. Ecological Monographs, 59, 1 - 20.

Caceres, C.E. (1997). Temporal variation, dormancy, and coexistence: a field test of the storage effect. Proceedings of the National Academy of Sciences of the United States of America, 94, 9171-9175.

Caswell, H. (2001). Matrix Population Models: Construction, Analysis, and Interpretation. 2nd edn. Sinauer Associates, Sunderland, MA.

Chesson, P. (1989). A general model of the role of environmental variabiltiy in communities of competing species. In: Some Mathematical Questions in Biology: Models in Population Biology. American Mathematical Society, Providence, RI, vol. 20 of Lectures on Mathematics in the Life Sciences, pp. 97-123.

Chesson, P. (1994). Multispecies competition in variable environments. Theoretical Population Biology, 45, 227-276. 
Chesson, P. (2000a). General theory of competitive coexistence in spatially-varying environments. Theoretical Population Biology, 58, 211-237.

Chesson, P. (2000b). Mechanisms of maintenance of species diversity. Annual Review of Ecology and Systematics, pp. 343-366.

Chesson, P. (2003). Quantifying and testing coexistence mechanisms arising from recruitment fluctuations. Theoretical Population Biology, 64, 345 - 357.

Chesson, P. (2008). Quantifying and testing species coexistence mechanisms. In: Unity in Diversity: Reflections on Ecology after the Legacy of Ramon Margalef (eds. Valladares, F., Camacho, A., Elosegui, A., Estrada, M., Gracia, C., Senar, J. \& Gili, J.). Fundacio BBVA, Bilbao, pp. 119 164.

Chesson, P., Pacala, S. \& Neuhauser, C. (2001). Environmental niches and ecosystem functioning. In: The Functional Consequences of Biodiversity (eds. Kinzig, A.P., Pacala, S.W. \& Tilman, D.). Princeton University Press, Princeton NJ, no. 33 in Monographs in Population Biology, chap. 10, pp. 213-245.

Chesson, P. \& Warner, R. (1981). Environmental variability promotes coexistence in lottery competitive systems. American Naturalist, 117, 923-943.

Chu, C. \& Adler, P. (2015). Large niche differences emerge at the recruitment stage to stabilize grassland coexistence. Ecological Monograph, 85, 373 - 392.

Coulson, T. (2012). Integral projections models, their construction and use in posing hypotheses in ecology. Oikos, 121, 1337-1350.

Descamps-Julien, B. \& Gonzalez, A. (2005). Stable coexistence in a fluctuating environment: an experimental demonstration. Ecology, 86, 2815 - 2824.

Dewi, S. \& Chesson, P. (2003). The age-structured lottery model. Theoretical Population Biology, $64,331-343$.

Ellner, S.P., Childs, D.Z. \& Rees, M. (2016). Data-driven Modeling of Structured Populations: A Practical Guide to the Integral Projection Model. Springer, New York. 
Ellner, S.P. \& Rees, M. (2006). Integral projection models for species with complex demography. American Naturalist, 167, 410-428.

Ellner, S.P. \& Rees, M. (2007). Stochastic stable population growth in integral projection models. Journal of Mathematical Biology, 54, 227-256.

Fan, J. \& Gijbels, I. (1996). Local Polynomial Modelling and Its Applications. Chapman \& Hall, London.

Freckleton, R.P., Watkinson, A.R. \& Rees, M. (2009). Measuring the importance of competition in plant communities. Journal of Ecology, 97, 379-384.

Mathias, A. \& Chesson, P. (2013). Coexistence and evolutionary dynamics mediated by seasonal environmental variation in annual plant communities. Theoretical Population Biology, 84, 56-71.

Metcalf, C.J.E., Ellner, S.P., Childs, D.Z., Salguero-Gómez, R., Merow, C., McMahon, S.M., Jongejans, E. \& Rees, M. (2015). Statistical modelling of annual variation for inference on stochastic population dynamics using Integral Projection Models. Methods in Ecology and Evolution, 6, $1007-1017$.

Pake, C. \& Venable, D. (1995). Is coexistence of sonoran desert annuals mediated by temporal variability in reproductive success. Ecology, 76, 246-261.

Shmida, A. \& Ellner, S. (1984). Coexistence of plant species with similar niches. Vegetatio, 58, $29-55$.

Smith, H.L. (1981). Competitive coexistence in an oscillating chemostat. SIAM Journal of Applied Mathematics, 40, $498-522$.

Smith, H.R. \& Waltman, P. (1995). The Theory of The Chemostat: Dynamics of Microbial Competition. vol. 13 of Cambridge Studies in Mathematical Biology. Cambridge University Press, Cambridge.

Sommer, U. (1984). The paradox of the plankton: Fluctuations of phosphorus availability maintain diversity of phytoplankton in flow-through cultures. Limnology and Oceanography, 29, 633 - 636 . 
Sommer, U. (1985). Comparison between steady state and non-steady state competition: Experiments with natural phytoplankton. Limnology and Oceanography, 30, 335-346.

Stewart, F.M. \& Levin, B.R. (1973). Partitioning of resources and the outcome of interspecific competition: a model and some general considerations. The American Naturalist, 107, 171-198.

Teller, B.J., Adler, P.B., Edwards, C.B., Hooker, G. \& Ellner, S.P. (2016). Linking demography with drivers: climate and competition. Methods in Ecology and Evolution, 7, 171 - 183.

Tuljapurkar, S. (1990). Population Dynamics in Variable Environments. Springer-Verlag, New York.

Usinowicz, J., Wright, S.J. \& Ives, A.R. (2012). Coexistence in tropical forests through asynchronous variation in annual seed production. Ecology, 93, 2073-2084.

Yuan, C. \& Chesson, P. (2015). The relative importance of relative nonlinearity and the storage effect in the lottery model. Theoretical Population Biology, 105, 39-52.

Yuan, C. \& Chesson, P. (2016). A structured lottery model for species coexistence in a variable environment: general coexistence mechanisms for species with complex life histories. In review. 


\begin{tabular}{|c|c|}
\hline Notation & Meaning or formula \\
\hline$n_{j}(t)$ & Population state of species $i$ at time $t$. In an IPM this is short for $n_{j}(z, t)$. \\
\hline$N_{j}(t)$ & Total population measure of species $j$ at time $t$ (total number, total biomass, etc.) \\
\hline$E_{j}(t)$ & Environment-dependent parameter (or parameter vector) for species $j$. \\
\hline \multirow[t]{2}{*}{$C_{j}(t)$} & $\begin{array}{l}\text { Competition experienced by species } j \text {. This must be a function of populations and } \\
\text { environment, }\end{array}$ \\
\hline & $C_{j}(t)=c_{j}\left(E_{1}(t), n_{1}(t), E_{2}(t), n_{2}(t), \cdots, E_{M}(t), n_{M}(t)\right)$ \\
\hline$r_{j}(t)$ & $\begin{array}{l}\text { and can be a vector of competition pressures on different vital rates or life-stages. } \\
\text { Instantaneous population growth rate, } r_{j}(t)=\log \left(N_{j}(t+1) / N_{j}(t)\right) \text { in discrete } \\
\text { time, and } r_{j}(t)=\frac{1}{N_{i}} \frac{d N_{j}}{d t} \text { in continuous time. }\end{array}$ \\
\hline \multirow[t]{2}{*}{$K_{j}$} & $\begin{array}{l}\text { Projection matrix or kernel for species } j \text { in a matrix model or IPM. It must be } \\
\text { possible to write } K_{j} \text { as a function } K_{j}\left(E_{j}(t), C_{j}(t), \theta\right) \text { where } \theta \text { is a vector of constant } \\
\text { model parameters. So for each species, }\end{array}$ \\
\hline & $n_{j}(t+1)=K_{j}\left(E_{j}(t), C_{j}(t), \theta\right) n_{j}(t)$ \\
\hline$j \backslash k$ & $\begin{array}{l}\text { A value for species } j \text {, when species } k \text { is absent from the community and all other } \\
\text { species are present. }\end{array}$ \\
\hline $\bar{r}_{j}$ & Average value of $r_{j}$ in a simulation of the model, $\bar{r}_{j}=\mathbb{E}\left[r_{j}\left(E_{j}(t), C_{j}(t)\right)\right]$ \\
\hline$r_{j}^{\#}$ & $\begin{array}{l}\text { Average value of } r_{j} \text { using } C_{j} \text { from a baseline simulation and a second, independent } \\
\text { realization of the environment process } E_{j}^{\#}, r_{j}^{\#}=\mathbb{E}\left[r_{j}\left(E_{j}^{\#}(t), C_{j}(t)\right)\right] .\end{array}$ \\
\hline$q_{i r}$ & Scaling factors in the between-species measure of the storage effect. \\
\hline
\end{tabular}

\section{Box 1: Summary of notation used in the paper.}


1. Define environment $E$ and competition $C$, and write the competition model in terms of them. Then for each species $i$ as invader in turn, carry out the following steps (all $C_{j}$ below are $C_{j \backslash i}$, similarly for all $r_{j}$ ).

2. Generate and save environmental sequences $E_{j}(t)$ and $E_{j}^{\#}(t)$ for each species $j=1, \cdots, M$ for $t=0,1 \cdots, T$. Alternatively, if using empirical data or the original $E_{j}(t)$ series is deterministic, obtain $E_{j}^{\#}(t)$ by randomly shuffling $E_{j}(t)$, using the same shuffling for all species to preserve between-species correlations.

3. Do a simulation using the $E \mathrm{~s}$, computing and saving the competition parameters $C_{j}$ for all $j$, including $i$ (if using empirical data, calculate the $C_{j}(t)$ from the measured $E_{j}(t) \mathrm{s}$ and population densities). Then do a second simulation (or second calculation of population growth rates from experimental data) using the $E^{\#}$ s with the $C$ s from the first simulation. At each time step of each simulation, compute the population growth rates $r_{j}(t)$ and $r_{j}^{\#}(t)$. For structured population, the calculations of $r_{j}^{\#}(t)$ should use the population structure time series from the first simulation (or the actual experiment).

4. Compute the average population growth rates $\bar{r}_{j}=\mathbb{E}\left[r_{j}(t)\right], r_{j}^{\#}=\mathbb{E}\left[r_{j}^{\#}(t)\right]$. Note that if $\bar{r}_{i}=r_{i}^{\#}$ and $\bar{r}_{r}=r_{r}^{\#}$ regardless of which species is the invader, there is no storage effect in the system.

5. Calculate the scaling factors $q_{i j}$ using one of the following methods (ranked from most preferable to least):

(a) Analytic derivation using eqn. (15). See Section SI.5(a) for an example.

(b) Compute and save $\mathscr{C}_{i}$ and $\mathscr{C}_{j}$ during the model simulations, and fit a regression to estimate $q_{i j}$, as described in the text below eqn. (15) and in Section SI.5(b).

(c) Use the scaling factors for models with a common limiting factor, eqn. (SI.19), with one of the $\mathscr{C}_{r}$ as the limiting factor, as explained in Section SI.5(c).

(d) Use (eqn. SI.19) by perturbing the population size (at all size classes in a structured model), as described in the text around eqn (SI.23) and in Section SI.5(d).

6. Calculate the storage effect using eqns. (8) (2 species) or (19) (>2 species).

Box 2: Steps for calculating the storage effect for species $i$ in a community of $M \geq 2$ competing species. 
Table 1: Components of the storage effect for coexisting diatoms (Descamps-Julien \& Gonzalez, 2005). Subscripts $i$ and $r$ refer to the species in invader and resident states. $\chi$ denotes the covariance between $E$ and $C \cdot \bar{r}, r^{\#}$ and $r^{b}$ indicate, respectively, average population growth rates in baseline simulations, simulations with $E C$ covariance removed, and simulations with silicate concentration $S$ held constant. Both species necessarily have $\bar{r}_{r}=0$. Source files: ForcedChemoSubs.R, ForcedChemo_rbars_Deltas.R

\begin{tabular}{ccccccccc}
\hline & $\bar{r}_{i}$ & $r_{i}^{\#}$ & $r_{r}^{\#}$ & $\chi_{i}$ & $\chi_{r}$ & $\Delta I_{b}$ & $r_{i}^{b}$ & $r_{r}^{b}$ \\
\hline Fragillaria & 0.061 & 0.058 & 0.00057 & -0.035 & 0.0099 & 0.042 & 0.24 & 0.0012 \\
Cyclotella & 0.007 & 0.005 & 0.034 & -0.018 & 0.17 & 0.0029 & 0.0058 & 0.16 \\
\hline
\end{tabular}


Table 2: Invasion growth rate $\bar{r}_{i}$ and the storage effect contribution $\Delta I_{b}$ (in parentheses) for the empirical IPM. "All" means the fitted model which has variability in survival, growth and recruitment. The other columns are results with variability in only one component, holding the coefficients in other components constant at their mean. Values of 0 indicate an estimate $<0.001$ in magnitude. The results are based on simulations of 5000 generations, with the first 500 discarded so that the system was in steady state during the time period used for estimation. Five replicates were done for each simulation (defined by which vital rate(s) varied, and which species was invading). Standard errors for each estimate in the Table are given in Section Section SI.8. Source files: IPM-empirical-wrapper.r, IPM-empirical-summary.r and scripts that they source.

\begin{tabular}{lllll}
\hline Species & All & Survival & Growth & Recruitment \\
\hline Artemisia tripartita & $0.017(0)$ & $-0.016(0)$ & $0.018(0)$ & $0.023(0)$ \\
Hesperostipa comata & $0.164(0.002)$ & $0.130(-0.012)$ & $0.130(-0.012)$ & $0.089(0)$ \\
Poa secunda & $0.360(-0.010)$ & $0.332(-0.002)$ & $0.332(-0.001)$ & $0.222(0)$ \\
Pseudoroegneria spicata & $0.169(0.001)$ & $0.133(0.002)$ & $0.134(0.002)$ & $0.084(0)$ \\
\hline
\end{tabular}




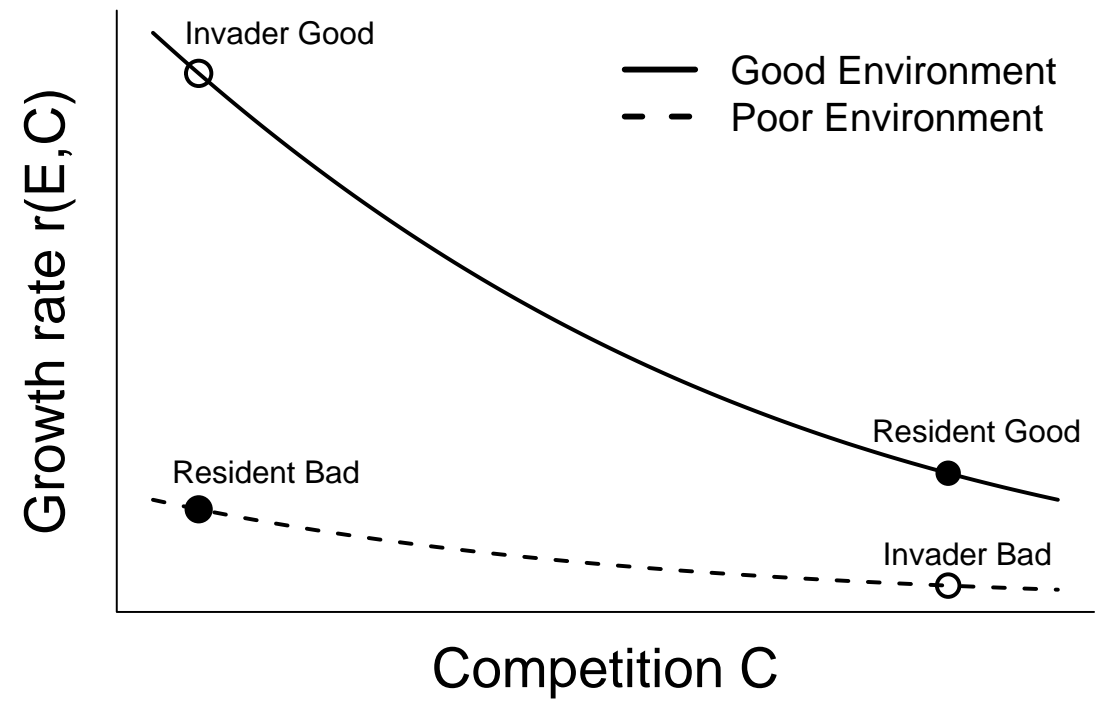

Figure 1: An illustration of how EC covariance and subadditivity can produce the storage effect. The labelled points show population growth rates when $E C$ covariance affects a resident more than an invader. When the resident has a good year, the competition it experiences is high, so the resident has only moderately good population growth. When the invader has a good year, the competition that it experiences is nonetheless low (because the invader is rare, and the resident is either having a bad year or does not compete much with the invader), so the invader has high population growth rate. Because of subadditivity, the invader's gains in good years are much greater than the losses suffered in bad years. 

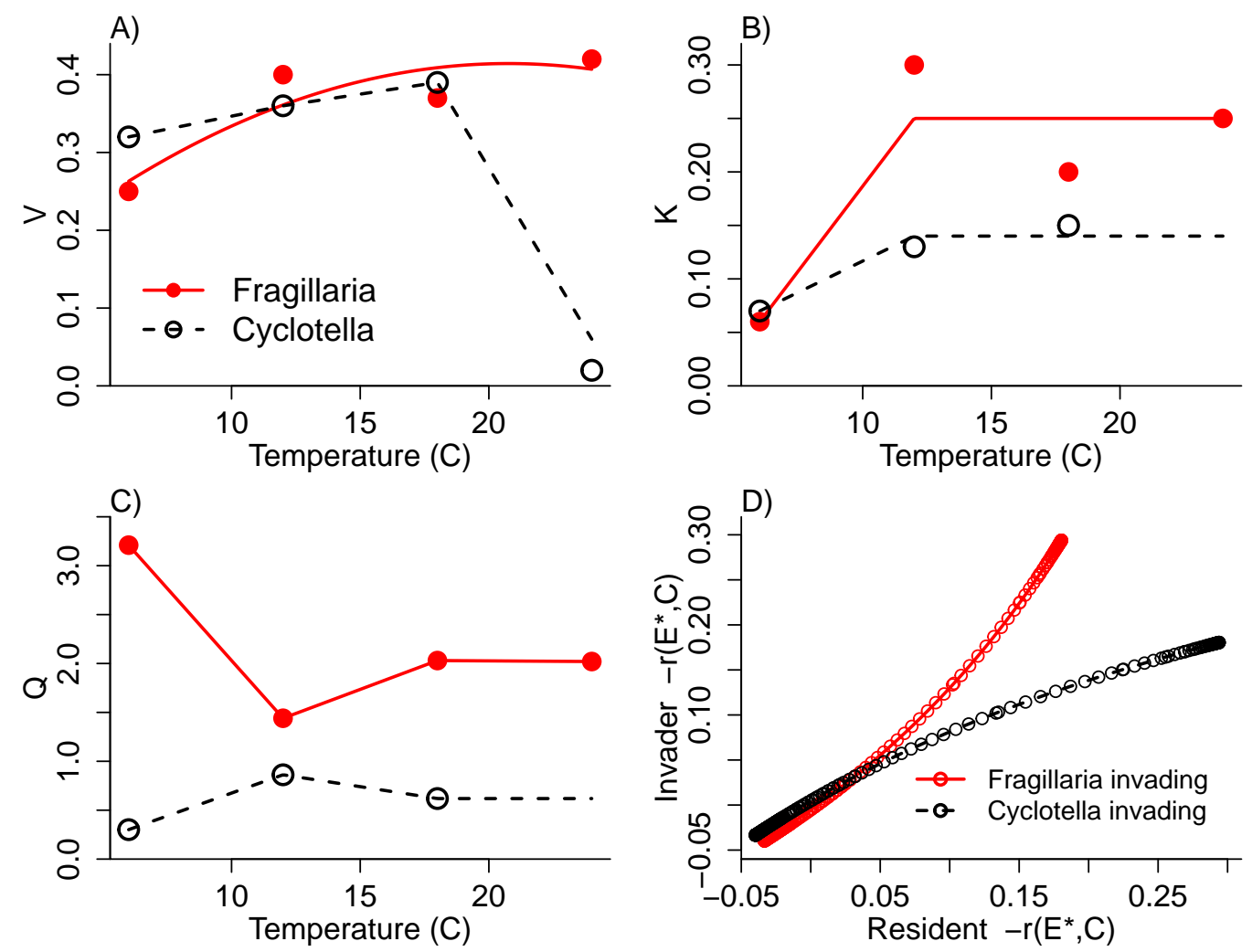

Figure 2: A),B),C) The species-specific temperature responses of the parameters $V, K$, and $Q$ governing nutrient uptake and conversion efficiency give rise to a storage effect, which we quantified through simulations of the chemostat model (10). Points (closed circles: Fragillaria, open circles: Cyclotella) are estimated values from 9 day batch experiments, Table 1 of Descamps-Julien \& Gonzalez (2005). The fitted lines and curves were used to simulate the model with continuously varying temperature. D) Plot of competition impacts on the invader, $\mathscr{C}_{i 1}$, versus competition impacts on the resident, $\mathscr{C}_{r_{1}}$, during two long model simulations with one species invading and the other resident; this is used to estimated the scaling factors $q_{i r}$. Note that $K$ and $Q$ for Cyclotella could not be estimated at $24^{\circ} \mathrm{C}$ because of its very low growth rate in the batch experiments. Cyclotella's growth at $24^{\circ} \mathrm{C}$ was much better in chemostats than in the batch experiments that the estimates plotted here are based on. Our $V$ function for Cyclotella (dashed line in panel A) therefore used a higher value of $V$ at $24^{\circ} \mathrm{C}$, chosen to make the model match better the average abundance of Cyclotella in chemostat experiments; even without this adjustment the model predicted coexistence of the two species in the variable temperature regime. Source files: ForcedChemoSubs.R, PlotForcedChemo.R, ForcedChemo_qir_regression.R 

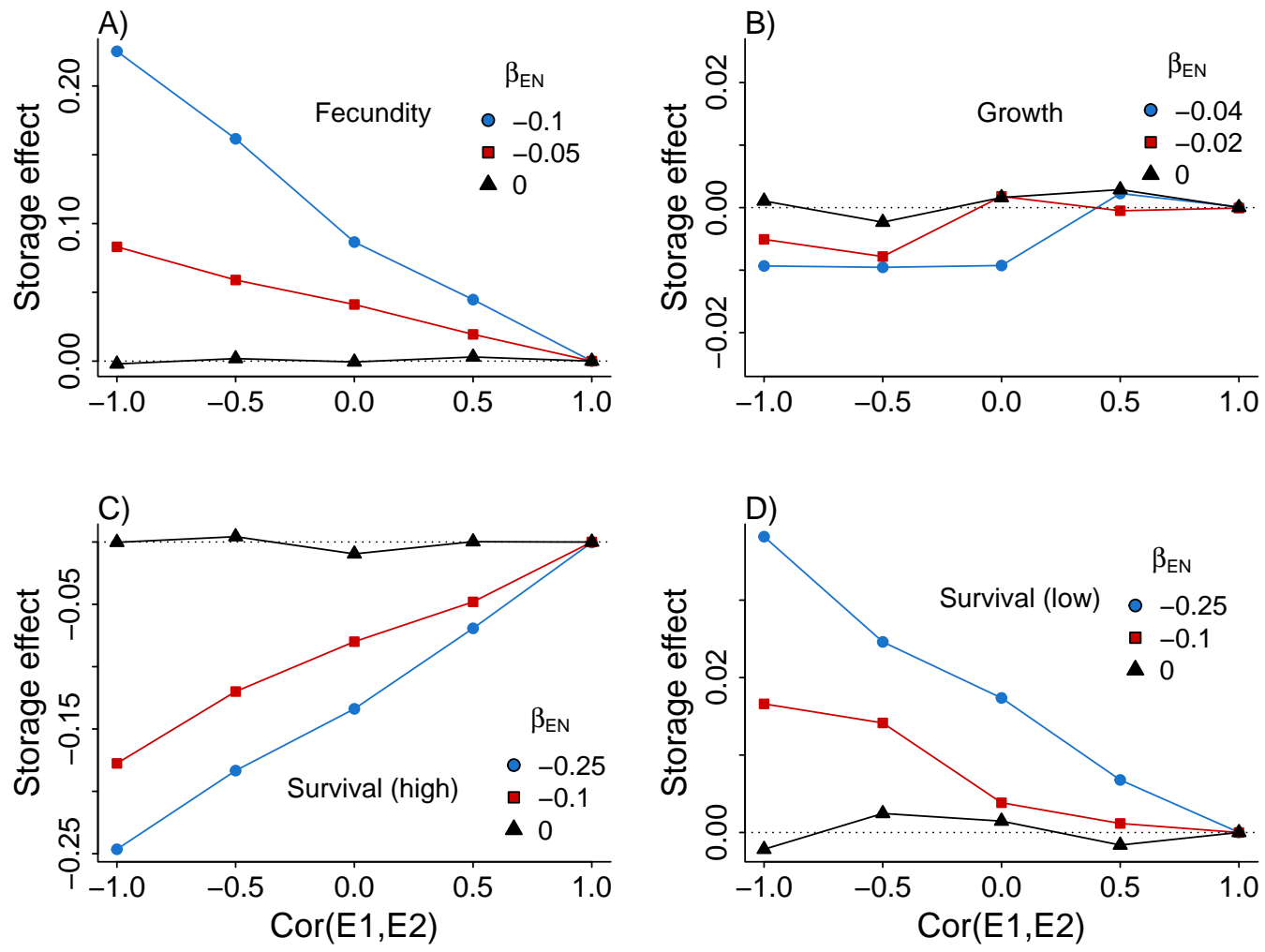

Figure 3: Results for the prototype IPM with symmetric parameters, as described in the text. In each panel, the environment covariates $E_{1}, E_{2}$ affect only the one vital rate noted in the figure; for all other rates the $E_{j}$ are held at zero (their mean value). Each panel shows the estimated storage effect $\Delta I_{b}$ (which has the same value for both species) as a function of the correlation between $E_{1}(t)$ and $E_{2}(t) ; \beta_{E N}$ in panel legends is the common value of all nonzero $\beta_{i j}$, and determines the strength of the environment by competition interaction. The storage effect cannot operate when $\beta_{E N}=0$. Source files: IPM-experiments-wrapper.R and scripts that it sources. 


\section{Supporting Information Appendix S1}

Ellner et al., "How to quantify the temporal storage effect..."

509

\section{Section SI.1 Additional Table and Figures}

Table SI-1: $\mathrm{R}$ code to compute storage effect for the lottery model with equal death rates. The same code with more extensive comments is in SI file LotteryCalculateDeltaIb. $R$

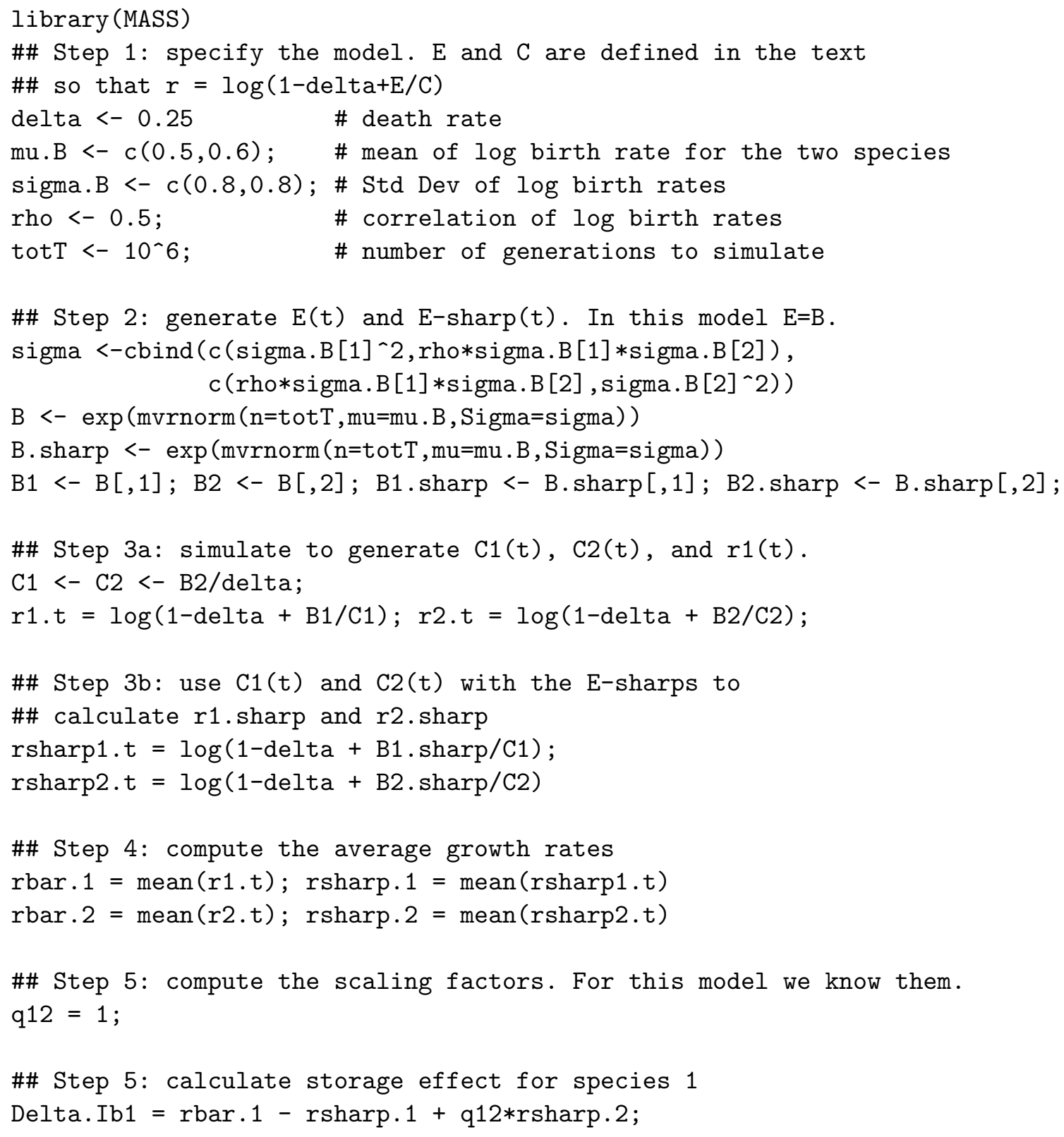



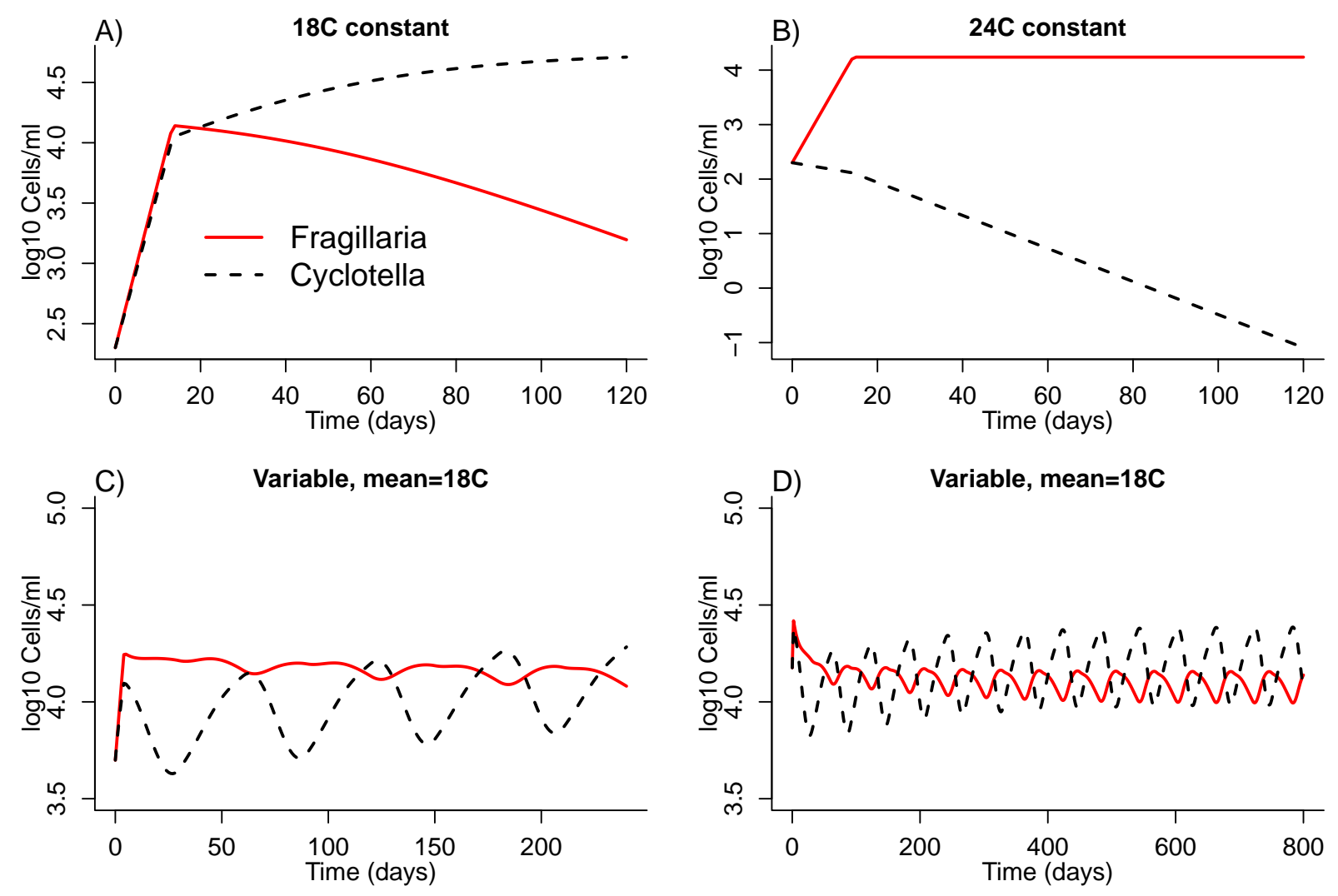

Figure SI-1: Simulation results for the Descamps-Julien \& Gonzalez (2005) chemostat model, confirming that the model matches the experimental observation that coexistence occurs under the fluctuating temperature regime (mean $24^{\circ} \mathrm{C}$, amplitude $6^{\circ} \mathrm{C}$, period 60 days) but only one species persists at either 18 or $24^{\circ} \mathrm{C}$ constant temperature. Source files: PlotForcedChemo.R, ForcedChemoSubs.R 

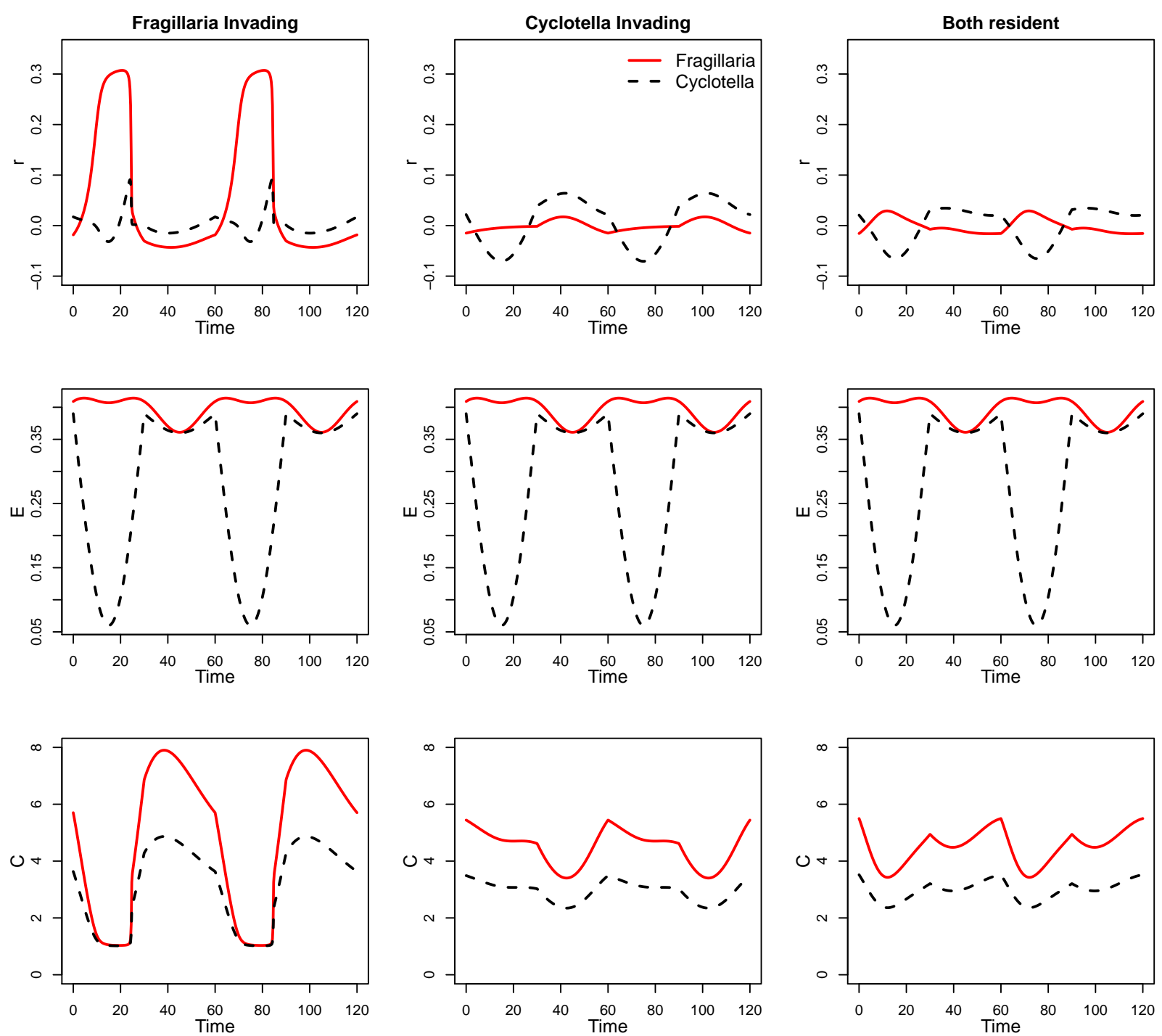

Figure SI-2: Simulation results for the Descamps-Julien \& Gonzalez (2005) chemostat model. The three columns show simulation results for Fragillaria invading Cyclotella at steady state, for $C y$ clotella invading Fragillaria at steady state, and for the coexistence steady state, over two complete cycles of the temperature variation (120 days). Top panels show the instantaneous population growth rates $r$ for the two species, middle panels show the time-varying environment parameter $E=$ $V$ (this is the same in all columns, because $V$ is determined strictly by temperature) and competition $C$ as defined in the main text. Source files: ForcedChemo_PlotInvasions.R,ForcedChemoSubs.R 


\section{Section SI.2 Standard parameters}

The analytic theory (Chesson, 1994) begins by transforming the environment and competition parameters $(E$ and $C)$ for each species to the "standard parameters" $\mathscr{E}$ and $\mathscr{C}$,

$$
\mathscr{E}=g\left(E, C^{*}\right), \quad \mathscr{C}=-g\left(E^{*}, C\right),
$$

where $E^{*}, C^{*}$ are baseline values of $E$ and $C$ (central values such as the mean or median) that are used in the analytic theory as the point about which Taylor expansions are done to derive small variance approximations. In this paper we mostly work with $E$ and $C$. Here we explain why that is legitimate.

There are two properties that we define in terms of standard parameters: subadditivity, and $E C$ covariance. The definition of subadditivity in terms of standard parameters is that $\frac{\partial^{2} \tilde{r}}{\partial \mathscr{E} \partial \mathscr{C}}<0$, where $\tilde{r}$ denotes $r$ as a function of $\mathscr{E}$ and $\mathscr{C}$. Our definition, equation (1), uses $E$ and $C$. But these two definitions are equivalent. Because $g$ is monotonic by assumption in each of its arguments, there are functions $h_{1}, h_{2}$ such that $\mathscr{E}=h_{1}(E), \mathscr{C}=h_{2}(C)$, both monotonic increasing. Then

$$
\frac{\partial r}{\partial E}=\frac{\partial}{\partial E} \tilde{r}\left(h_{1}(E), h_{2}(C)\right)=h_{1}^{\prime}(E) \frac{\partial \tilde{r}}{\partial \mathscr{E}}\left(h_{1}(E), h_{2}(C)\right) .
$$

Now differentiate both sides with respect to $C$, to see that the two definitions are equivalent because $h_{1}^{\prime} h_{2}^{\prime}>0$.

"EC covariance" refers to effects of the fact that $E$ and $C$ are not independent (in the probability theory sense of independence). The between-species storage effect measure, which we study in this paper, is the part of the difference between invader and scaled resident population growth rates that goes away if the covariance of $\mathscr{E}$ and $\mathscr{C}$ is set to 0 , while the marginal distributions of $\mathscr{E}$ and $\mathscr{C}$ are left the same (see p. 240 in Chesson (1994)). In our approach, we make $E$ and $C$ independent, while the marginal distributions of $E$ and $C$ remain the same. But because $\mathscr{E}$ is a function of $E$ alone, and $\mathscr{C}$ is a function of $C$ alone (recall that the baseline values are constants), our approach is exactly equivalent to making $\mathscr{E}$ and $\mathscr{C}$ independent (so their covariance is 0) while leaving their marginal distributions the same.

\section{Section SI.3 The community average storage effect measure}

The community average storage effect measure (Angert et al. (2009, SI eqn. 6), Chesson (2008, Table 6.3)) is a weighted sum of terms that compare each species in invader and resident states. We refer to the term for species $j$ as the "within-species" measure $\Delta I_{w, j}$, defined as follows. Define $\bar{r}_{j, I}=\bar{r}_{j \backslash j}$ as the mean population growth rate of species $j$ as an invader into the community, and (as in the main text) $\bar{r}_{j \backslash k}$ as the mean growth rate of species $j$ as a resident within the community (at stochastic steady-state) when species $k \neq j$ is absent. In a community of $M$ competing species, 
the within-species measure of storage effect for species $j$ is the contribution of $E C$ covariance to

$$
\begin{gathered}
\bar{r}_{j, I}-\bar{r}_{j, R}, \\
\text { where } \quad \bar{r}_{j, R}=\frac{1}{M-1} \sum_{k \neq j} \bar{r}_{j \backslash k} .
\end{gathered}
$$

In $\bar{r}_{j, I}$, species $j$ is an invader into a community of $M-1$ resident species. In $\bar{r}_{j, R}$, species $j$ is one resident in a community of $M-1$ residents, and we average over all such possible communities. Note that there are no scaling factors $q_{i r}$; this is because the community average measure results from a weighted average of the between-species measures $\Delta I_{b, j}$ such that the scaling factors cancel out (see Chesson, 2003, 2008).

In the community average measure, the invader and resident in Figure 1 are the same species, at low and high frequency in the community. The difference $\bar{r}_{j, I}-\bar{r}_{j, R}$ represents the gain (or loss) in population growth rate as a result of becoming rare. We measure storage effect by asking: how much of this change in population growth rate is due to the storage effect? Because the storage effect is the result of $E C$ covariance, an equivalent question is: how much of $\bar{r}_{j, I}-\bar{r}_{j, R}$ is due to the change in $E C$ covariance when a species becomes rare?

To introduce the procedures, consider a two-species community. As with the between-species measure, the simulation steps are to

- generate the independent environment sequences $E_{1}(t), E_{2}(t)$ and $E_{1}^{\#}(t), E_{2}^{\#}(t)$.

- do a long "baseline" model simulation with species 1 as the invader

- at each time step compute and save the population growth rate $r_{1, I}(t), r_{2, R}(t)$ of the two species, and the corresponding growth rates $r^{\#}(t)$ that result from replacing each $E_{j}(t)$ by $E_{j}^{\#}(t)$, retaining everything else from the baseline simulations. As before, average the saved growth rates (omitting an initial burn-in period) to compute the estimates

$$
\bar{r}_{1, I}=\mathbb{E}\left[r_{1, I}(t)\right], \quad r_{1, I}^{\#}=\mathbb{E}\left[r_{1, I}^{\#}(t)\right], \quad \bar{r}_{2, R}=\mathbb{E}\left[r_{2, R}(t)\right], \quad r_{2, R}^{\#}=\mathbb{E}\left[r_{2, R}^{\#}(t)\right]
$$

- To compute $\bar{r}_{2, I}, \bar{r}_{1, R}$ and the corresponding "sharped" population growth rates, repeat the entire process with species 1 as the resident, and species 2 invading.

The within-species measure of storage effect for species $j$ is then

$$
\Delta I_{w, j}=\left(\bar{r}_{j, I}-\bar{r}_{j, R}\right)-\left(r_{j, I}^{\#}-r_{j, R}^{\#}\right)=\bar{r}_{j, I}-r_{j, I}^{\#}+r_{j, R}^{\#}, j=1,2 .
$$

Computing $\bar{r}$ and $r^{\#}$ for all species during a single simulation is important when there are more than 2 species. If environment series $E(t)$ and $E^{\#}(t)$ are generated for all species before any simulations are run, then one model simulation with species $j$ invading and all other species resident can be used to calculate $\bar{r}_{j, I}, \bar{r}_{k \backslash j}$ for all $k \neq j$, and all of the corresponding "sharped" population 
growth rates for each species. $M$ simulations, one with each species invading, then provide all of the $\bar{r}$ and $r^{\#}$ values needed to compute $\Delta I_{w}$ for all species using equations (SI.3a) and (SI.3b).

\section{Section SI.4 Comparison of simulation and analytic approaches for the symmetric two-species lottery model}

Here we compare our simulation-based measure of the storage effect $\Delta I_{b}$ to the formulas in Chesson (1994) for the case of small fluctuations in fecundity in the symmetric two-species lottery model with equal death rates. This example illustrates that our approach is equivalent to previous analytic theory without the additional small-variance assumptions that the analytic theory requires, by showing that if you first apply our approach and then add to it the small-variance assumptions, the published analytic formula is recovered.

It is convenient to switch to the Chesson (1994) definitions in which the environment parameter $E$ is the $\log$ of per-capita fecundity, $b_{i}(t) \equiv \log B_{i}(t)$, and the competition parameter $C$ is the $\log$ of the ratio between the total number of juveniles and the number of open sites,

$$
C_{i}(t)=\log \left(\frac{B_{1}(t) N_{1}(t)+B_{2}(t) N_{2}(t)}{\delta N}\right)
$$

This has no effect at all on our approach, because generating $B_{i}^{\#}(t)$ directly is exactly equivalent to generating $b_{i}^{\#}(t)$ and defining $B_{i}=e^{b_{i}}$. For species 1 invading species 2, equation (5) then becomes:

$$
\begin{aligned}
\bar{r}_{1} & =\mathbb{E} \log \left(1-\delta+\delta \exp \left(b_{1}-b_{2}\right)\right) \\
r_{1}^{\#} & =\mathbb{E} \log \left(1-\delta+\delta \exp \left(b_{1}^{\#}-b_{2}\right)\right) \\
r_{2}^{\#} & =\mathbb{E} \log \left(1-\delta+\delta \exp \left(b_{2}^{\#}-b_{2}\right)\right)
\end{aligned}
$$

Chesson (1994) derives the small-variance approximation to $\Delta I_{b}$ for the symmetric case where the species have equal mortality rates $\delta$, the $b_{i}$ have equal variance $\sigma^{2}$ and correlation $\rho$, so that $\operatorname{Cov}\left(b_{1}, b_{2}\right)=\rho \sigma^{2}$ :

$$
\Delta I_{b} \approx \sigma^{2} \delta(1-\delta)(1-\rho)
$$

For this symmetric case with equal death rates, the scaling factors are $q_{i r}=1$ (Chesson, 1994, Table 1), so in our approach $\Delta I_{b, 1}=\bar{r}_{1}-r_{1}^{\#}+r_{2}^{\#}$ in a simulation where species 1 is invading and species 1 resident. $b_{i}$ and $b_{i}^{\#}$ are two independent realizations of the same stochastic process, so we can simplify the calculations by noting that $b_{1}^{\#}-b_{2}$ has the same distribution as $b_{1}-b_{2}^{\#}$, and $b_{2}^{\#}-b_{2}$ has the same distribution as $b_{2}-b_{2}^{\#}$. We therefore have

$$
\begin{aligned}
& r_{1}^{\#}=\mathbb{E} \log \left(1-\delta+\delta \exp \left(b_{1}-b_{2}^{\#}\right)\right) \\
& r_{2}^{\#}=\mathbb{E} \log \left(1-\delta+\delta \exp \left(b_{2}-b_{2}^{\#}\right)\right) .
\end{aligned}
$$


In MAPLE we set

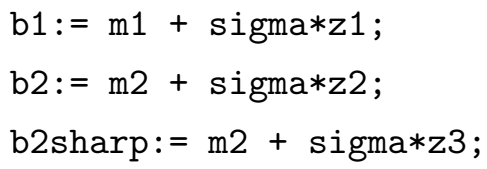

where the $z_{i}$ represent random fluctuations with mean 0 , variance $1 ; z_{1}$ and $z_{2}$ have correlation $\rho$, and $z_{3}$ is independent of $z_{1}$ and $z_{2}$. To approximate the expectations in (SI.6) we define

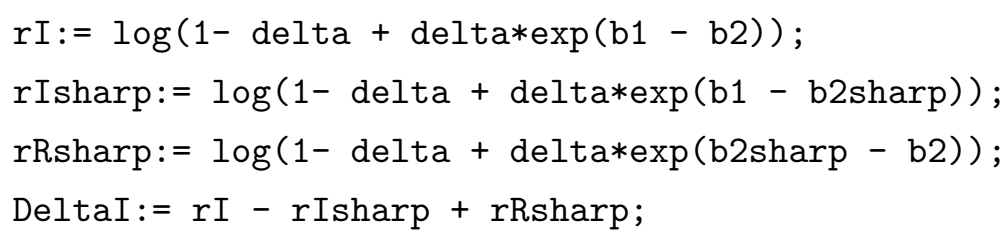

and do a Taylor explansion of DeltaI in $\sigma$ to order $\sigma^{2}$. We find that

- The constant (order 0) term is zero, as it should be.

- The order- $\sigma$ term has zero mean, as it should.

The order $\sigma^{2}$ term is:

$$
\begin{aligned}
& 1 / 2 \frac{\delta \mathrm{e}^{m_{1}-m_{2}}(z 1-z 2)^{2}}{1-\delta+\delta \mathrm{e}^{m_{1}-m_{2}}}-1 / 2 \frac{\delta^{2}\left(\mathrm{e}^{m_{1}-m_{2}}\right)^{2}\left(z_{1}-z_{2}\right)^{2}}{\left(1-\delta+\delta \mathrm{e}^{m_{1}-m_{2}}\right)^{2}} \\
& -1 / 2 \frac{\delta \mathrm{e}^{m_{1}-m_{2}}(z 1-z 3)^{2}}{1-\delta+\delta \mathrm{e}^{m_{1}-m_{2}}}+1 / 2 \frac{\delta^{2}\left(\mathrm{e}^{m_{1}-m_{2}}\right)^{2}\left(z 1-z_{3}\right)^{2}}{\left(1-\delta+\delta \mathrm{e}^{m_{1}-m_{2}}\right)^{2}} \\
& +1 / 2 \delta\left(z_{2}-z_{3}\right)^{2}-1 / 2 \delta^{2}\left(z_{2}-z_{3}\right)^{2} .
\end{aligned}
$$

We need to find the expectation of this expression. The properties of the $z_{j}$ imply that $\mathbb{E}\left(z_{1}-z_{2}\right)^{2}=$ $2(1-\rho), \mathbb{E}\left(z_{i}-z_{3}\right)^{2}=2$. Substituting these into the expression above, and using MAPLE to simplify, gives

$$
\text { Our } \Delta I_{b} \approx \sigma^{2} \delta(1-\delta)\left[1-\frac{\rho \mathrm{e}^{m_{1}-m_{2}}}{\left(1-\delta+\delta \mathrm{e}^{m_{1}-m_{2}}\right)^{2}}\right]
$$

This is qualitatively what we expect: the storage effect is maximized at intermediate $\delta$, high variance, and low correlation between resident and invader Es. In several cases our results agree with Chesson's formula (SI.7):

- When $\rho=0$, our result becomes $\sigma^{2} \delta(1-\delta)$, agreeing with Chesson's formula with $\rho=0$.

- Setting $m_{1}=m_{2}$ (equal mean fecundity for the two species) before doing the Taylor expansion, the result is again $\sigma^{2} \delta(1-\delta)(1-\rho)$, agreeing with Chesson's formula.

But when the species have unequal mean fecundity, we do not replicate (SI.7).

Reconciling our results with Chesson (1994) requires one more aspect of the small variance approximation used to derive (SI.7): "competitive differences between species are of similar magnitude to the means and variances of environmental fluctuations" (Chesson, 1994, p. 237). In the 
symmetric model this means that $m_{1}-m_{2}$ is $O(\sigma)$ or smaller. With this additional assumption, Taylor expansion shows that

$$
\frac{e^{m_{1}-m_{2}}}{\left(1-\delta+\delta e^{m_{1}-m_{2}}\right)^{2}}=1+O(\sigma)
$$

and therefore to order $\sigma^{2}$, our $\Delta I_{b}=\sigma^{2} \delta(1-\delta)(1-\rho)$.

In summary, when our simulation-based definition of $\Delta I_{b}$ is combined with the small variance assumptions used in Chesson (1994), we recover exactly Chesson's results for the symmetric lottery model with equal death rates.

\section{Section SI.5 More details about computing the scaling factors}

All approaches to computing the scaling factors start with the competition effects defined in equation (14), which we repeat here:

$$
\mathscr{C}_{j}=-r_{j}\left(E_{j}^{*}, C_{j}\right)
$$

The baseline environment $E_{j}^{*}$ is typically a central value of $E_{j}(t)$, such as the mean or median, but this is not a requirement. The scaling factors $q_{i r}$ that appear in the storage effect measure for species $i$ are calculated from the competition effects

$$
\mathscr{C}_{j \backslash i}=-r_{j}\left(E_{j}^{*}, C_{j \backslash i}\right)
$$

when species $i$ is invading (i.e., at zero or negligibly low density).

We now explain in detail each of the possible approaches for computing the scaling factors, from most to least preferable as listed in Box 2 of the main text.

\section{(a) Analytic calculation}

Scaling factors should be calculated analytically whenever this is possible. The analytic calculation approach can be used whenever an explicit and unique formula can be found for $\mathscr{C}_{i \backslash i}$ as a function of the effects $\mathscr{C}_{r \backslash i}, r=1,2, \cdots, M, r \neq i$ for the resident species. This is most likely to occur when there are only two species and the functional form of the model is relatively simple and does not involve transcendental functions.

One extremely simple example is the two-species symmetric lottery model. In that model, $C_{1}(t) \equiv C_{2}(t)$ : both are equal to the ratio between the total number of juveniles, and the total number of open sites. If we choose $E_{1}^{*}=E_{2}^{*}$ (which is possible because $E_{1}$ and $E_{2}$ have the same marginal distributions), then $\mathscr{C}_{2} \equiv \mathscr{C}_{1}$ at all times, and under all circumstances. The general formula for the scaling factors, equation (15), then states that

$$
q_{12}=\frac{\partial \mathscr{C}_{1 \backslash 1}}{\partial \mathscr{C}_{2 \backslash 1}}=\frac{d \mathscr{C}_{2 \backslash 1}}{d \mathscr{C}_{2 \backslash 1}}=1
$$

and for the same reason $q_{21}=1$. 


\section{(b) Simulation-regression approach}

The next-best situation is when there is a deterministic relationship between the competition experienced by the invader and the competition experienced by the resident species, but it cannot be found analytically (e.g., multispecies models whose dynamic equations include transcendental functions). The analysis in Chesson (1994) uses his assumption (a6), which states that the competitive impact $\mathscr{C}_{i \backslash i}$ experienced at any time by species $i$ as an invader, can be expressed as a function of the competitive impacts experienced by the resident species at the same time, $\left\{\mathscr{C}_{r \backslash i}\right\}$. So long as that is true, the scaling factors are defined by equation (15), and the functional relationship among the $\mathscr{C} \mathrm{s}$ can often be estimated by a regression analysis of simulation output, as follows.

The first step is to compute the time series of competitive impacts $\mathscr{C}_{j \backslash i}(t)$ for each species $j=1,2, \cdots, M$. In unstructured population models like our chemostat case study, there is a generally a formula for $r$ that can be used to compute $\mathscr{C}$ for each species at each time step of a simulation, directly from the definition (14). With structured populations, the population growth rate depends on population structure, so $\mathscr{C}_{j}(t)$ is computed by changing $E_{j}(t)$ to $E_{j}^{*}$ while retaining everything else, including the population structure at time $t$. In an IPM this means recomputing the kernel for species $j$ at time $t$ with $E_{j}^{*}$ in place of $E_{j}(t)$ and everything else the same (including $C_{j}(t)$, even if $C_{j}$ depends on $E_{j}$ ), applying the new kernel to the population state of species $j$ at time $t$ in the simulation, and recording the change in log total population size (or total cover, etc.) between time $t$ and time $t+1$. The population state at $t+1$ computed using $E_{j}^{*}(t)$ is discarded, because the simulation continues from the population state computed using $E_{j}(t)$.

Then, having generated values of $\mathscr{C}_{j \backslash i}$ for each species in a simulation with species $i$ invading (or absent), the partial derivative in (15) can be evaluated by doing a regression of $\mathscr{C}_{i \backslash i}$ on $\left\{\mathscr{C}_{r \backslash i}\right\}$. This is a simple regression if there is only one resident (a two-species community) and multiple regression with more than one resident. If the relationship is linear, the slope coefficients in the linear regression are then the $q_{i r}$ for invading species $i$ and all of the residents. If the relationship is nonlinear, the slope of the fitted nonlinear regression at the point where $\mathscr{C}_{r \backslash i}=0$ for all residents should be used, because this is the point about which the Taylor expansion of invader growth rate is done in the small-variance analytic theory.

This process has to be repeated $M$ times for an $M$ species community, once with each species as the invader to compute the scaling factors that figure into the $\Delta I_{b}$ for that species.

The two-species lottery model with unequal death rates is a simple example where we can verify that the simulation-regression approach to estimating the $q_{i r}$ leads to the same result as the analytic theory when environmental variance is small. This example illustrates the fact that the simulationregression approach is equivalent to the analytic approach without requiring any additional smallvariance assumptions. Without loss of generality we can let species 1 be the invader, and species 2 resident. In the notation of the main text, which is more convenient for this analysis, $E_{j}(t)=B_{j}(t)$ 
and the two species experience the same competition $C_{1}(t)=C_{2}(t)=B_{2}(t) / \delta_{2}$. We then have

$$
\mathscr{C}_{j}(t)=-r_{j}\left(E_{j}^{*}, C(t)\right)=-\log \left(1-\delta_{j}+E_{j}^{*} / C(t)\right), j=1,2 .
$$

Over the course of a simulation, variation over time in $C(t)$ will produce values for $\mathscr{C}_{1}(t)$ and $\mathscr{C}_{2}(t)$ that can be plotted against each other, as we did for the two species in the chemostat case study in Fig. 2D. However, because $C$ is always the same for both species, we can calculate analytically the slope of the regression function:

$$
\frac{\partial \mathscr{C}_{1}}{\partial \mathscr{C}_{2}}=\frac{\partial \mathscr{C}_{1} / \partial C}{\partial \mathscr{C}_{2} / \partial C}=\frac{E_{1}^{*}\left(1-\delta_{2}+E_{2}^{*} / C\right)}{E_{2}^{*}\left(1-\delta_{1}+E_{1}^{*} / C\right)}
$$

As in Section SI.4, formula (SI.16) is reconciled with Chesson (1994) when we apply the same small-variance assumptions. In the small-variance analysis, $q_{12}$ is the value of (SI.16) at the baseline values $E_{j}^{*}$ and $C_{j}^{*}$. Chesson (1994) chooses to use a common baseline value of $C$, so $C_{1}^{*}=C_{2}^{*}=C^{*}$. The baseline $E$ values are then determined by the requirement that $r_{j}\left(E_{j}^{*}, C_{j}^{*}\right)=0$, implying that

$$
E_{j}^{*} / C^{*}=\delta_{j}
$$

Substituting (SI.17) into (SI.16) we get $q_{12}=\delta_{1} / \delta_{2}$ and by symmetry $q_{21}=\delta_{2} / \delta_{1}$, exactly the same as Chesson (1994, Table 1).

There are some potential complications to the simulation-regression approach. First, the assumed function relating invader and resident $\mathscr{C} \mathrm{s}$ might not exist. However, the regression analysis can still be used to calculate the $q_{i r}$ based on the expected value of $\mathscr{C}_{i \backslash i}$ conditional on $\left\{\mathscr{C}_{r \backslash i}\right\}$, which is the closest analog to what the $q_{i r}$ accomplish under assumption (a6) in Chesson (1994). ${ }^{4}$ The $q_{i r}$ are chosen to remove from $\Delta C$ any effect of mean response to competition, to the order of accuracy of the small fluctuations approximation. Starting from equation (21) in Chesson (1994) we have

$$
\Delta C=\mathbb{E}\left[\mathscr{C}_{i \backslash i}-\sum_{r} q_{i r} \mathscr{C}_{r \backslash i}\right]=\mathbb{E}\left[\mathbb{E}\left(\mathscr{C}_{i \backslash i}-\sum_{r} q_{i r} \mathscr{C}_{r \backslash i}\right) \mid\left\{\mathscr{C}_{r \backslash i}\right\}\right]
$$

$$
=\mathbb{E}[\overbrace{\mathbb{E}\left[\mathscr{C}_{i \backslash i} \mid\left\{\mathscr{C}_{r \backslash i}\right\}\right]}^{1}-\overbrace{\sum_{r} q_{i r} \mathscr{C}_{r \backslash i}}^{(2)}] .
$$

The $q_{i r}$ are defined so that the linear terms cancel out when we Taylor-expand terms (1) and (2) in (SI.18), as functions of the $\left(C_{r \backslash i}-C_{r}^{*}\right)$, to second order around 0. Under assumption (a6) of Chesson (1994), $\mathscr{C}_{i \backslash i}$ is a deterministic function of $\left\{\mathscr{C}_{r \backslash i}\right\}$, and definition (15) causes the linear term in (1) - (2) to be identically zero. Without assumption (a6) this is impossible, but we can still make the linear term equal zero in expectation, so that it still contributes zero to $\Delta C$. This will be true

\footnotetext{
${ }^{4}$ To follow the rest of this paragraph, you need to have read Chesson (1994) at least up to the end of section 4. Your other option is skipping to the next paragraph below, taking it on trust that the simulation-regression approach is appropriate in this situation.
} 
if the $q_{i r}$ are the coefficients in the linear approximation to $\mathbb{E}\left[\mathscr{C}_{i \backslash i} \mid\left\{\mathscr{C}_{r \backslash i}\right\}\right]$ as a function of $\left\{\mathscr{C}_{r \backslash i}\right\}$. That is exactly what is estimated by the simulation-regression approach.

\section{(c) Models with a common limiting factor}

A more difficult complication for the simulation-regression approach is that there may be several different functions relating invader and resident $\mathscr{C} \mathrm{s}$ (or their expectations), so that the $q_{\text {ir }}$ are not uniquely defined. There is then not a unique linear approximation that can be estimated by the regression method, and as a result the regression method fails for reasons we explain below in the paragraph containing equation (SI.22). Non-uniqueness arises unavoidably if several resident species are responding to a single limiting factor. The result is near-perfect collinearity among the $\mathscr{C}_{r \backslash i}(t)$ vectors. In such a situation, scaling factors are not uniquely defined because $\mathscr{C}_{i}$ can be written as a function of any one of the collinear $\mathscr{C}_{r}$, or any combination of them. In such cases, Chesson (1994, p. 255) suggests that the scaling factors should be defined in a way that "treats the resident species in an equivalent manner", which leads to the following recipe (Chesson, 1994, p. 251). Define one of the $\mathscr{C}_{r \backslash i}$ to be the limiting factor $F$ for species $i$ as invader, and do univariate nonlinear regressions (as in the previous subsection) to estimate how the other $\mathscr{C}$ s (or their expectations) depend on $F$,

$$
\mathbb{E}\left[\mathscr{C}_{k \backslash i}(t)\right]=\phi_{k, i}(F(t)), k=1,2, \cdots, M .
$$

The scaling factors are then

$$
q_{i r}=(1 /(M-1)) \phi_{i, i}^{\prime} / \phi_{r, i}^{\prime}
$$

with the derivatives evaluated at a central value of $F$ (e.g., the mean or median value, in the simulation with species $i$ invading).

\section{(d) Perturbation approach}

There is no corresponding recipe for more complicated kinds of collinearity, or other causes for non-uniqueness of a function giving $\mathscr{C}_{i \backslash i}$ as a function of the $\left\{\mathscr{C}_{r \backslash i}\right\}$. For example, it does not cover a situation where each species responds (in a different way) to the same two limiting factors. The effect of a non-unique relationship is that estimates of the $q_{i r}$ will be very sensitive to small random perturbations of the predictors, so that small changes in model parameters, or a different seed for the random number generator, could easily lead to very different estimates of $q_{i r}$. Another likely outcome, which we encountered in our empirical IPM case study, is that estimated $q_{i r}$ can be negative. To understand how nonuniqueness leads to negative $q_{i r}$ consider the hypothetical case of a common limiting factor $Z$, for species 1 invading species 2 and 3 , with

$$
\mathscr{C}_{1}=Z-1, \mathscr{C}_{2}=2 Z-2, \mathscr{C}_{3}=3 Z-3 .
$$


We have $\phi_{1}^{\prime}=1, \phi_{2}^{\prime}=2, \phi_{3}^{\prime}=3$ and the recipe (SI.19) gives $q_{12}=1 / 4, q_{13}=1 / 6$. This corresponds to the fact that

$$
\mathscr{C}_{1}=\frac{1}{4} \mathscr{C}_{2}+\frac{1}{6} \mathscr{C}_{3}
$$

because applying the definition $q_{i r}=\frac{\partial \mathscr{C}_{i}}{\partial \mathscr{C}_{r}}$ to (SI.21) we get $q_{12}=1 / 4, q_{13}=1 / 6$. But it is also true that

$$
\mathscr{C}_{1}=5 \mathscr{C}_{2}-3 \mathscr{C}_{3}
$$

which leads to $q_{12}=5, q_{13}=-3$; and also $\mathscr{C}_{1}=3 \mathscr{C}_{3}-4 \mathscr{C}_{2}$ giving $q_{12}=-4, q_{13}=3$, and so on. So if the relationship between $\mathscr{C}_{i}$ and the $\mathscr{C}_{r}$ is non-unique, it is easy for the regression approach to give estimated $q$ s that are large and opposite in sign. This is what we obtained for several species using the regression approach on the empirical IPM. A negative $q_{i r}$ is not necessarily a conceptual problem. It means that in computing $\Delta I_{b}$, a mechanism that increases the population growth rate of resident $r$ is counted as contributing to invader $i$ population growth, and that may be reasonable if that resident facilitates growth of the invader. The problem here, however, is different: a negative estimate of $q_{i r}$ when in fact the species are competing for a common limiting resource.

In this case and others where the definition (15) cannot be applied, recent results for a structured population model suggest that it is reasonable to instead calculate scaling factors using (SI.19) with the total abundance of all stages (or individual states) within all species as the limiting factor (P.L. Chesson, personal communication), as follows.

The ratio $\phi_{i, i}^{\prime} / \phi_{r, i}^{\prime}$ intuitively represents the relative sensitivity of the species to an increase in competition. This can be estimated by perturbing competition, and seeing how much each species changes in population growth rate. Competition is perturbed by making the same small increase in the density of all categories within every resident species (but not the invader). In an IPM this means perturbing $n_{j}(z, t)$ to $n_{j}(z, t)+\epsilon$ for all $z$ in every resident species. With unstructured populations, this is just adding $\epsilon$ to the total population size of each species. As in our "sharped" simulations with structured population models, the only change is the addition of $\epsilon$, and everything else (including the population structure time series) is carried over from the baseline simulation. For each time step in the baseline simulation, the value of $C_{j}(t)$ for each species is recomputed using the perturbed populations, and population growth rate is recomputed. In an IPM this means recomputing the kernels for each species using the recomputed $C(t)$ values, applying the recomputed kernels to the population structure at that time in the baseline simulation, and recording the population growth rate that results. Let $\widetilde{r}_{j}$ denote the time-average of these population growth rates with perturbed $C(t)$. The scaling factors are then estimated as

$$
q_{i r}=(1 /(M-1)) \frac{\bar{r}_{i}-\widetilde{r}_{i}}{\bar{r}_{r}-\widetilde{r}_{r}}
$$

We caution readers that (SI.23) is based on generalizing from the analysis of one simple structured model with two discrete life stages. Further analysis of structured population models should 
soon either firm up or modify the recommendations. For now, we recommend that that whenever possible, the $q_{i r}$ should be derived analytically for the model at hand, or calculated by the multiple regression approach when the relationship among the $\mathscr{C} \mathrm{s}$ is identifiable.

\section{Section SI.6 Subadditivity of $r$ for the prototypical IPM}

We consider here the situation in Figure 3 of the main text, in which only one of the vital rates (survival, growth, or fecundity) is fluctuating in response to a varying environment variable $E(t)$. Our question is, when do we have subadditivity (equation 1) so that storage effect can operate and promote coexistence, and when do we have the opposite inequality so that storage effect opposes coexistence?

Total number of individuals and total cover have the same long-term growth rates (Tuljapurkar, 1990; Ellner \& Rees, 2007) so we can define $r$ in terms of total cover $\int e^{z} n(z) d z$ (as we do in the main text) rather than total number of individuals. Let $\tilde{n}$ denote the current population structure normalized to have total cover 1 ; then the instantaneous growth rate in total cover is $r(E, C)=\log \lambda(E, C)$ where

$$
\lambda=\langle u, K(E, C) \tilde{n}\rangle, \quad u(z)=e^{z}
$$

and $\langle a, b\rangle$ denotes the inner product $\int a(z) b(z) d z$.

Basic calculus applied to eqn. (SI.24) gives

$$
\frac{\partial^{2} r}{\partial E \partial C}=\frac{1}{\lambda}\left\langle u, \frac{\partial^{2} K}{\partial E \partial C} \tilde{n}\right\rangle+\frac{-1}{\lambda^{2}}\left\langle u, \frac{\partial K}{\partial E} \tilde{n}\right\rangle\left\langle u, \frac{\partial K}{\partial C} \tilde{n}\right\rangle
$$

$\left\langle u, \frac{\partial K}{\partial E} \tilde{n}\right\rangle$ is positive, because larger $E$ in any of the vital rate models results in more individuals or larger individuals at the next time step, and $\left\langle u, \frac{\partial K}{\partial C} \tilde{n}\right\rangle<0$ because higher $C$ has the opposite effect. The second term on the right-hand side of (SI.25) is therefore always positive, opposing subadditivity of $r$ and making a negative contribution to storage effect.

The sign of the first right-hand term depends on which one of the vital rates is fluctuating in response to $E$. When it is either survival and fecundity, the entries in the kernel $K=s G+B$ are linear functions of a response $R$ (survival probability, or per-capita offspring number) of the form $R=f\left(b_{0}+b_{1} z+b_{2} E-C\right)$ where $f$ is the inverse of the link function in the regression model. $\frac{\partial^{2} K}{\partial E \partial C}$ therefore has the sign of $\frac{\partial^{2} R}{\partial E \partial C}=-b_{2} f^{\prime \prime}\left(b_{0}+b_{1} z+b_{2} E-C\right)$.

The inverse link function for fecundity is $f(x)=e^{x}$ with $f^{\prime \prime}>0$ so $\frac{\partial^{2} K}{\partial E \partial C}<0$ and the first term on the right-hand side of (SI.25) is negative. A positive contribution of storage effect is possible if the first term outweighs the second, and this occurs for the parameters used in Fig. 3.

The inverse link function for survival is $f(x)=e^{x} /\left(1+e^{x}\right)$. This has $f^{\prime \prime}>0$ when $x<0$ corresponding to survival probability below 0.5 , so storage effect can be positive, but $f^{\prime \prime}<0$ for $x>0$ corresponding to survival probability above 0.5 , so storage effect must be negative. Both of these match our results in Fig. 3. 
Growth is more complicated because $K$ is (all else being equal) proportional to a Gaussian function of $b_{2} E-C$, hence the partial derivatives in (SI.25) all take both positive and negative values within the range of the integrations that calculate the inner product. However, a different approach shows that $r$ is approximately additive in $E$ and $C$ when environmental variability affects only growth. An individual at the initial time who survives to size $z^{\prime}$ at the subsequent time has cover $e^{z^{\prime}}$. $z^{\prime}$ has mean $\mu(z)=b_{0}+b_{1} z+b_{2} E-C$ and size-independent variance which we can represent as a random variable $\varepsilon$, writing $z^{\prime}=\mu(z)+\varepsilon$. The total cover of survivors at the subsequent time is therefore $\mathbb{E}\left[s(z) e^{\mu(z)+\varepsilon}\right]$ where $\mathbb{E}$ here is joint expectation over $\tilde{n}$ (the initial distribution of $z$ ) and the growth variability $\varepsilon$ (recall that we are here studying $r$ as a function of $E$ and $C$, rather than $r$ as a random variable driven by variation in $E$ and $C$ ). In our toy IPM, as in the empirical IPM that it is loosely based on, new recruits are very small and contribute little to the total cover in the subsequent year. If we ignore their contribution, then $\lambda$ is the total cover of survivors:

$$
\lambda \approx \mathbb{E}\left[s(z) e^{b_{0}+b_{1} z+b_{2} E-C+\varepsilon}\right]=e^{\left(b_{2} E-C\right)} \mathbb{E}\left[s(z) e^{b_{0}+b_{1} z+\varepsilon}\right] .
$$

It follows that $r=\log \lambda$ is approximately equal to $b_{2} E-C$ plus a constant depending on the initial size distribution and the growth variance. Therefore $\frac{\partial^{2} r}{\partial E \partial C}=0$, neither sub- nor super-additive, so the storage effect due to variability in growth is approximately zero, as we found in our numerical results. As with survival and fecundity, this conclusion is a consequence of the link function in the demographic model (i.e., the fact that $\mu(z)$ is a linear function of $E$ and $C$ ).

\section{Section SI.7 Methods for the empirical IPM}

This section borrows heavily from the corresponding SI sections of Adler et al. (2010), because the model we use here is a generalized version of that model.

\section{Extracting demographic data from digitized quadrat maps}

Genets were classified as survivors or new recruits using a computer program that tracks genets based on their spatial locations within the quadrats (Lauenroth and Adler 2008). For example, when a genet present in year $t+1$ overlaps in space with a conspecific genet present in year $t$, we assume it to be the same genet. If a genet in year $t+1$ is more than $5 \mathrm{~cm}$ from any conspecific genet present in year $t$, we classify it as a recruit. Our approach allows genets to fragment and/or coalesce over the study period. Some plants were identified by the original mappers as seedlings; we classified these plants as recruits regardless of their location.

For parameterizing our models we represented each genet as a circle with area equal to the sum of all polygons in the map assigned to that genet, centered at the genet's centroid. Very small plants were originally mapped as points; we represented those as circles with an area of $0.25 \mathrm{~cm}^{2}$. The distance between two genets was defined to be the distance between their centroids. Information on the fate of plants located along quadrat edges was not used in the statistical modeling of growth and 
survival. However, edge plants were included in the amount of neighborhood crowding experienced by more centrally located genets.

\section{Statistical modeling of survival and growth}

We assume that the survival probability and growth of individual genets is a function of genet size, the neighborhood-scale crowding experienced by the genet, temporal variation among years, and permanent spatial variation among groups of quadrats (the 4-6 quadrats within each group are generally within $50 \mathrm{~m}$ of each other, while groups may be separated by up to $3 \mathrm{~km}$ ).

Our model for neighborhood crowding assumes that the influence of neighbors on a focal individual depends on the distance, $d$, to the neighbor and the neighbor's size, $u$ :

$$
w_{l j m, t}=\sum_{k} e^{-\alpha_{j m} d_{l j k m, t}^{2}} u_{k m, t}
$$

Here, $w_{l j m, t}$ is the crowding that genet $l$ in species $j$ in year $t$ experiences from neighbors of species $m, \alpha_{j m}$ determines the spatial scale over which neighbors of species $m$ exert influence on a genet of species $j, k$ indexes all the focal genet's neighbors of species $m$ at time $t$, and $d_{l j k m, t}$ is the distance between genet $l$ in species $j$ and genet $k$ in species $m$. Using squared distances implies a Gaussian competition kernel. An exponential kernel performed marginally better in the statistical models, but caused simulations of the individual-based model to crash. The total crowding impact on a genet was assumed to be a weighted sum of the impacts from each species,

$$
w_{l j, t}^{V}=\sum_{m=1}^{4}\left(\bar{\omega}_{j m}^{V}+\omega_{j m, t}^{V}\right) w_{l j m, t}
$$

where $V=S$ or $G$, indicating Survival or Growth. Note that the competition coefficients $\omega$ are different for survival and growth but the distance-weighted neighborhood crowding $w$ is the same, because the fitted values of $\alpha$ for survival and growth were similar enough that we assumed a common value. We estimated an average competition coefficient $\bar{\omega}$, and a time-varying competition coefficient $\omega_{j m, t}^{S}$ that was fitted as a random year effect.

We modeled the survival probability, $S$, of genet $l$ in species $j$ and group $g$ from time $t$ to $t+1$ as

$$
\operatorname{logit}\left(S_{l j g, t}\right)=\gamma_{j, t}^{S}+\phi_{j g}^{S}+\beta_{j, t}^{S} u_{l j, t}+w_{l j, t}^{S}
$$

where $\gamma$ is a time-dependent intercept, and $\phi$ is the coefficient for the effect of quadrat group. Fitting this model to the data included estimation of the average and year-specific competition coefficients $\bar{\omega}_{j m}^{S}$ and $\omega_{j m, t}^{S}$.

Our model for expected growth conditional on survival has a similar structure:

$$
\mathbb{E}\left[u_{i j g, t+1}\right]=\gamma_{j, t}^{G}+\phi_{j g}^{G}+\beta_{j, t}^{G} u_{i j, t}+w_{i j, t}^{G}+\epsilon_{i j, t}^{G}
$$


Following previous analyses of these data (Adler et al., 2010; Chu \& Adler, 2015) we modeled the variance in growth as a nonlinear function of predicted genet size:

$$
\operatorname{Var}\left(u_{l j g, t+1}\right)=a e^{b \mathbb{E}\left[u_{l j g, t+1}\right]} .
$$

\section{Statistical modeling of recruitment}

In contrast to survival and growth, which are modeled at the individual level, we model recruitment at the quadrat level because we cannot determine which recruits were produced by which potential parents. The model is a form of a Ricker equation for discrete time population growth. We assume that the number of individuals, $y$, of species $j$ recruiting at time $t+1$ in location $q$ follows a negative binomial distribution (the observations appeared overdispersed relative to a Poisson model):

$$
y_{j q, t+1} \sim \operatorname{NegBin}\left(\lambda_{j q, t+1}, \theta\right)
$$

where $\lambda$ is the mean and $\theta$ is the size parameter. In turn, $\lambda$ depends on the composition of the quadrat in the previous year :

$$
\lambda_{j q, t+1}=C_{j q, t}^{\prime} e^{\left(\gamma_{t}^{R}+\phi_{g}^{R}+\omega_{t}^{R} C_{q t}^{\prime}\right)}
$$

$C_{j q t}^{\prime}$ is the cover $\left(\mathrm{cm}^{2}\right)$ of species $j$ in quadrat $q$ at time $t, \gamma$ is a time-dependent intercept, $\phi$ is a coefficient for the effect of group location, $\omega$ is a vector of time-varying coefficients that determine the strength of intra- and interspecific density-dependence; the year-specific $\omega_{j k, t}^{R} \mathrm{~s}$ (e.g. the effect of species $k$ on species $j$ at time $t$ ) are drawn from a normal distribution with mean $\bar{\omega}_{j k}^{R}$ and variance $\sigma_{j k}^{R}$, which are themselves drawn from a prior distribution with a mean of zero and large variance. $C^{\prime}$ is the vector of effective cover of each species. By estimating each species' effective cover in a quadrat, we recognized that plants outside the mapped quadrat may contribute recruits to the focal quadrat, and vice versa. We estimated effective cover in a quadrat $q$ as a mixture of the observed cover in the focal quadrat and the mean cover across the group $g$ in which the quadrat is located:

$$
C_{j q t}^{\prime}=p_{j} C_{j q t}+\left(1-p_{j}\right) \bar{C}_{j g t},
$$

where $p$ is the mixing fraction between 0 and 1 .

\section{Parameter estimation}

Adler et al. (2010) and Chu \& Adler (2015) conducted model selection analyses to determine which parameters should vary through time, whether size and crowding interact, and whether values of $\alpha$ should vary with the focal species, the neighbor species, or both. Here we retain the model structures of Chu \& Adler (2015) and simply add random year effects on competition.

Parameters of each model were estimated in a Bayesian framework using WinBUGS 1.4 (Lunn et al. 2000) via the R2WinBUGS package, using exactly the same methods as Chu \& Adler (2015). Each model was run for 30,000 MCMC iterations of three chains with different initial values for 
parameters. We discarded the initial 10,000 MCMC samples, and the remaining samples were thinned to 1 out of every 20 time steps to reduce autocorrelation. Convergence of the three chains was verified using the Brook and Gelman potential scale reduction factor.

\section{Integral projection model}

In our IPM, the population of species $j$ is represented by a density function $n\left(u_{j}, t\right)$ which gives the density of genets of size $u$ at time $t$, with genet size on natural-log scale, i.e. $n\left(u_{j}, t\right) d u$ is the number of genets whose area (on arithmetic scale) is between $\exp \left(u_{j}\right)$ and $\exp \left(u_{j}+d u\right)$. The density function for size $v$ at time $t+1$ is given by

$$
n_{j}\left(v_{j}, t+1\right)=\int_{L_{j}}^{U_{j}} k_{j}\left(v_{j}, u_{j}, \bar{w}_{j}\left(u_{j}\right)\right) n_{j}\left(u_{j}, t\right)
$$

where the kernel $k_{j}$ describes all possible transitions from size $u$ to $v$ and $\bar{w}_{j}$ is a vector whose elements are the average crowding experienced by an individual of size $u_{j}$ in species $j$ from all species in the community. We describe below how $\bar{w}_{j}$ is calculated from the density functions for the species in the model. The integral is evaluated over a size interval $[L, U]$ that extends beyond the range of observed sizes.

The kernel is constructed from the fitted survival $(\mathrm{S})$, growth $(\mathrm{G})$, and recruitment $(\mathrm{R})$ models:

$$
k_{j}\left(v_{j}, u_{j}, \bar{w}_{j}\right)=S_{j}\left(u_{j}, \vec{n}\right) G_{j}\left(v_{j}, u_{j}, \vec{n}\right)+R_{j}\left(v_{j}, u_{j}, \vec{n}\right)
$$

where $\vec{n}$ is the set of size-distribution functions for all species in the community. $S$ is given by eqn. (SI.29) and $G$ by eqns. (SI.30) and (SI.31), using an expected neighborhood crowding calculated from the size distribution functions. In fitting the vital rate regressions, we calculated a neighborhood crowding unique to each individual $i$ based on the spatial locations and sizes of neighboring plants (eqn. SI.27). This spatially-explicit approach cannot be extended to the IPM, which does not track individual locations. Instead, we used spatially-implicit approximations that incorporate the essential features of local neighborhood competition. When we analyzed the spatial point patterns of conspecifics in the observed data, we found that while very small individuals were distributed randomly, large genets had a distribution that was more regular (Adler et al. 2010, Chu and Adler 2015). Thus, large plants experience less conspecific crowding than small plants on average. However, this pattern is much weaker for heterospecific spatial patterns.

For heterospecific crowding, we applied the simplest mean-field approximation, which assumes that plant locations (the centers of the circles representing individual genets) are distributed randomly and independently. In this approximation, (Adler et al., 2010) showed that the mean crowding exerted by species $k$ on a species $j$ individual is given by

$$
\bar{w}_{j k}=\frac{\pi N_{k} \bar{X}_{k}}{\alpha_{k} A}
$$


where $N$ is the average density of species $k$ (individuals per quadrat), $\bar{X}$ is the average size of species $k$ individuals (on absolute scale), $\alpha$ is the spatial scale over which species $k$ affects neighbors (defined in eqn. SI.27), and $A$ is the area of a quadrat, in the same units as $\bar{X}$.

The principal feature of the overdispersion of large plants is that conspecific large plants do not overlap. More specifically, large plants have very few conspecific neighbors closer than twice the mean radius of large plants of their species. For conspecifics, we therefore modified our mean-field approximation by assuming that plants are distributed at random subject to a "no-overlap" rule which requires that the centers of any two conspecific genets must be separated by at least the sum of their radii. With the no-overlap constraint, the mean conspecific crowding experienced by a species $j$ individual of radius $\mathrm{r}$ due to neighbors of species $k$ is given by

$$
\bar{w}_{j k}(r)=2 \pi \int_{r}^{\infty} z e^{-\alpha_{j k} z^{2}} C_{k}(z-r) d z
$$

where $C_{k}(z-r)$ is the total cover of plants of species $k$ of radius $z-r$ or smaller (Adler et al., 2010). When we simulated the IPM using eqn. (SI.38) for $k=j$ and eqn. (SI.37) for $k \neq j$, the model generated realistic abundances for all species.

For recruitment, the factor $\Phi=\exp \left(\gamma_{t}^{R}+\phi_{g}^{R}+\omega_{t}^{R} C_{q t}^{\prime}\right)$ in eqn (SI.32) gives the total cover of new recruits produced per quadrat, per unit area of potential parents. To incorporate this recruitment function into the IPM, we assumed that individual fecundity increases linearly with size, hence $R_{j}\left(v_{j}, u_{j}, \vec{n}\right)=c_{0, j}\left(v_{j}\right) e^{u_{j}} \Phi$ where $c_{0, j}$ is the initial size distribution of recruits. This has the consequence that recruitment by any species is proportional to total cover, as desired. $\Phi$ is calculated from $\vec{n}$ by converting the size distributions into total cover values, $C_{j}^{\prime}=\int e^{z} n_{j}(z, t) d z$. To see exactly how this all works, you can look at the code, which is available as online SI for this article. Un-zip the code file, and look in the StorageEffectEmpirical folder.

\section{Additional Literature cited}

Lauenroth, W. K., and P. B. Adler. 2008. Demography of perennial grassland plants: survival, life expectancy and life span. Journal of Ecology 96:1023-1032.

Lunn, D. J., A. Thomas, N. Best, and D. Spiegelhalter. 2000. WinBUGS - A Bayesian modelling framework: Concepts, structure, and extensibility. Statistics and Computing 10: 325-337.

R Core Team. 2015. R: A Language and Environment for Statistical Computing. R Foundation for Statistical Computing, Vienna, Austria.

Sturtz, S., U. Ligges and A. Gelman. 2005. R2WinBUGS: A Package for Running WinBUGS from R. Journal of Statistical Software 3: 1-16.

\section{Section SI.8 Additional simulation results for the empirical IPM}

. The values below are output from IPM-empirical-summary.r, copy-pasted in from an R terminal window to avoid transcription errors. These supplement the results in the main text (Table 1B) by 
giving the standard error for each $\bar{r}$ and storage effect estimate. The 4 rows of each matrix printed below refer to the four species in alphabetical order, as in Table 1B.

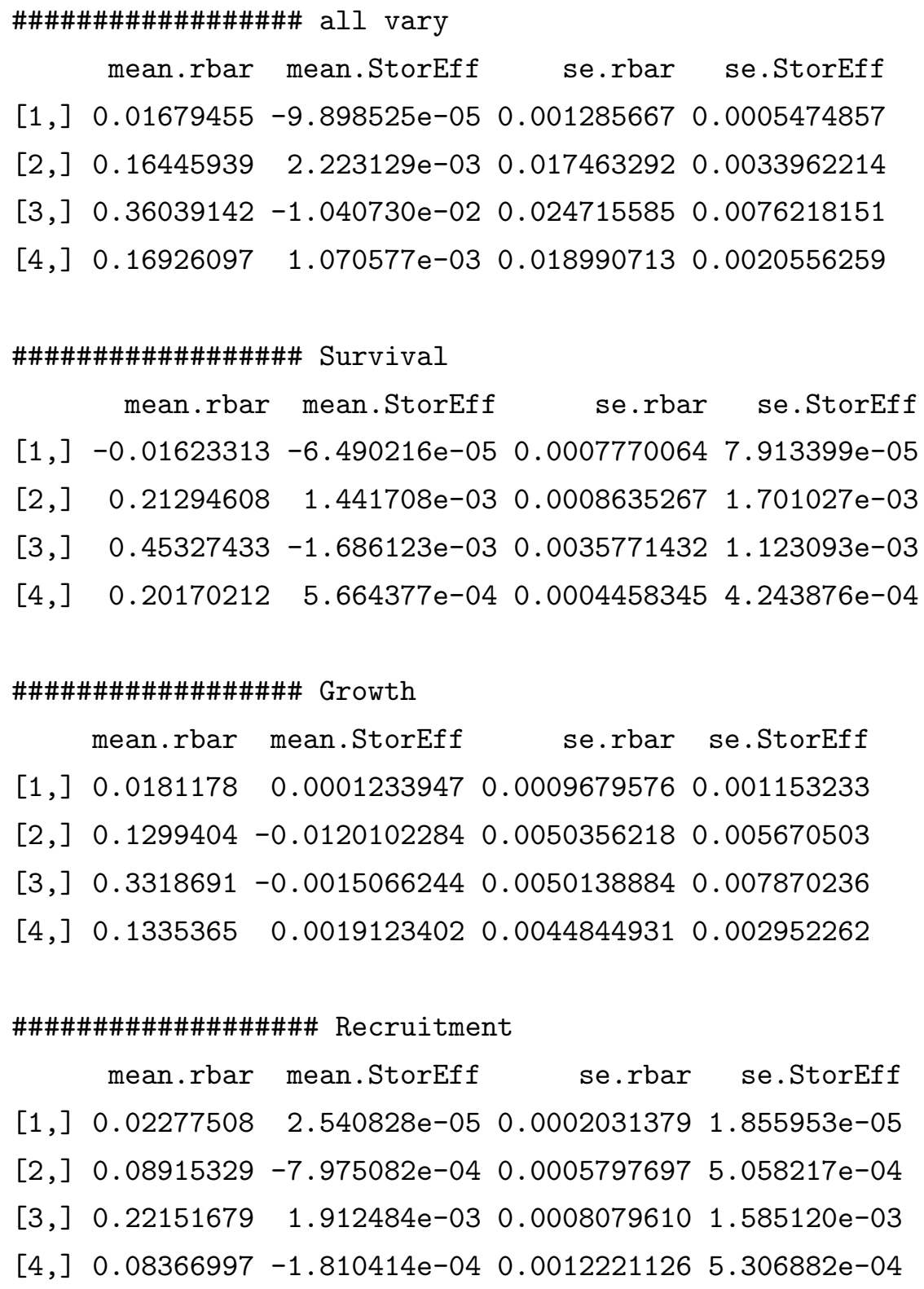

